\title{
Genomic characteristics and comparative genomics analysis of the endophytic fungus Sarocladium brachiariae
}

\author{
Yang Yang ${ }^{1}$, Xiaobao Liu', Jimiao Cai ${ }^{1}$, Yipeng Chen ${ }^{1}$, Boxun Li' ${ }^{1}$ Zhikai Guo ${ }^{2}$ and Guixiu Huang ${ }^{1 *}$
}

\begin{abstract}
Background: Sarocladium brachiariae is a newly identified endophytic fungus isolated from Brachiaria brizantha. A previous study indicated that $S$. brachiariae had antifungal activity; however, limited genomic information restrains further study. Therefore, we sequenced the genome of S. brachiariae and compared it with the genome of S. oryzae to identify differences between a Sarocladium plant pathogen and an endophyte.

Results: In this study, we reported a gapless genome sequence of a newly identified endophytic fungus Sarocladium brachiariae isolated from Brachiaria brizantha. The genome of $\mathrm{S}$. brachiariae is $31.86 \mathrm{Mb}$, with a contig N50 of $3.27 \mathrm{Mb}$ and 9903 protein coding genes. Phylogenomic analysis based on single copy orthologous genes provided insights into the evolutionary relationships of S. brachiariae and its closest species was identified as S. oryzae. Comparative genomics analysis revealed that S. brachiaria has 14.9\% more plant cell wall degradation related CAZymes to S. oryzae, and 33.3\% more fungal cell wall degradation related CAZymes, which could explain the antifungal activity of S. brachiaria. Based on Antibiotics \& Secondary Metabolite Analysis Shell (antiSMASH) analysis, we identified a contact helvolic acid biosynthetic gene cluster (BGC) for the first time in S. oryzae. However, S. brachiaria had seven fewer terpene gene clusters, including helvolic acid BGC, compared with S. onyzae and this may be associated with adaptation to an endophytic lifestyle. Synteny analysis of polyketide synthases (PKS), non-ribosomal peptide synthetases (NRPS), and hybrid (PKS-NRPS) gene clusters between S. brachiariae and S. oryzae revealed that just 37.5\% of tested clusters have good synteny, while 63.5\% have no or poor synteny. This indicated that the $S$. brachiariae could potentially synthesize a variety of unknown-function secondary metabolites, which may play an important role in adaptation to its endophytic lifestyle and antifungal activity.

Conclusions: The data provided a better understanding of the Sarocladium brachiariae genome. Further comparative genomic analysis provided insight into the genomic basis of its endophytic lifestyle and antifungal activity.
\end{abstract}

Keywords: Sarocladium brachiariae, Comparative genomics, CAZymes, Secondary metabolism, Gene cluster

\section{Introduction}

The Sarocladium genus was firstly established in 1976 based on two fungal pathogens causing sheath rot of rice [1]. Based on rDNA and internal transcribed spacer (ITS) sequences, some Acremonium species were recently relocated to the genus Sarocladium. Most Sarocladium species are plant and human pathogens, such as S. oryzae, S. kiliense, and S. strictum, and include a maize endophyte, $S$. zeae $[2,3]$. In previous study, we isolated an endophytic fungus strain HND5 from healthy

\footnotetext{
* Correspondence: hgxiu@vip.163.com

${ }^{1}$ Environment and Plant Protection Institute, Chinese Academy of Tropical

Agricultural Sciences, 4 Xueyuan Road, Haikou 571101, China

Full list of author information is available at the end of the article
}

leaves of Brachiaria brizantha collected form Danzhou China. This fungus has raised, cottony and moist to slimy colonies on PDA and produces branching conidiophore, cylindrical conidia arranged in slimy heads. Key morphological feature of the fungi is the production of hyphal coil. Together with ITS and LSU rDNA sequence phylogenetic analysis, we proposed it as Sarocladium brachiariae (MycoBank no. 814539), a new species of Sarocladium [4]. This new species prominently shows broad-spectrum inhibition to growth of many tested plant pathogenic fungi on solid media plate, including Colletotrichum gloeosporioides of mango trees, Fusarium oxysporium f.sp. cubense, Gloeosporium musarum, Colletotrichum gloeosporioides of rubber trees, Corynespora

(c) The Author(s). 2019 Open Access This article is distributed under the terms of the Creative Commons Attribution 4.0 International License (http://creativecommons.org/licenses/by/4.0/), which permits unrestricted use, distribution, and 
cassicola of papaya, Fusarium oxysporium of bamboo, Magnaporthe grisea, Bipolaris oryzae Shoem, Colletotrichum falcatum Went and Drechslera sp. We also observed S. brachiariae could colonize inside root of banana using GFP-tagging. And colonization of S. brachiariae could reduce the incidence of banana fusarium wilt in the field [5]. The genomic resources of Sarocladium in public database are limited and just whole genome of phytopathogenic species $S$. oryzae has been sequenced and published [6]. In order to unravel the genomic basis of endophytic lifestyle and antifungal activity of $S$. brachiariae, we sequenced the whole genome of $S$. brachiariae and made a comparative analysis with genome of S. oryzae.

Carbohydrate-active enzymes (CAZymes) are responsible for the breakdown, biosynthesis or modification of glycoconjugates, oligo- and polysaccharides [7]. Fungi can produce all kinds of CAZymes and Hittalmani et al. identified 1042 glycoside hydrolases (GHs), 1115 glycosyltransferases (GTs), 416 carbohydrate esterases (CEs), 270 auxillary activities (AAs) and 11 polysaccharide lyases (PLs) from genome of S. oryzae Saro-13 strain [6]. Secreted CAZymes involved in plant cell wall or fungal cell wall degradation received special attention because of their importance in phytopathogenic and endophytic fungi penetration of their hosts or biocontrol fungi inhibition of target pathogenic fungi. To overcome plant cell wall to colonize, plant pathogenic and endophytic fungi produce various enzymes to deconstruct cell well polysaccharides and these enzymes are called "cell walldegrading enzymes (CWEDs)" [8, 9]. The CAZy database (Carbohydrate Active Enzymes database, http://www. cazy.org/) has classified CWEDs and divided them into different families [7]. Enzymes involved in cellulose and hemicellulose hydrolysis are distribute mainly in the glycoside hydrolase $(\mathrm{GH})$ families [10]. As pectin degradation requires polygalacturonidases and pectin/pectate lyases, CWEDs involved in pectin hydrolysis are classified into GH 28 and polysaccharide lyases (PL) families [11]. In contrast to the plant cell wall, the fungal cell wall is mainly composed by chitin and $\beta-(1,3)$-glucan. Thus, secreted chitinases and $\beta-(1,3)$-glucanases are responsible for fungal cell wall degradation [12]. According to the CAZy classification, enzymes involved in chitin degradation mainly belong to $\mathrm{GH} 18$ and GH75 families, and the enzymes responsible for $\beta$ - $(1,3)$-glucan could be found in the GH55, GH16 and GH81 families $[10,13]$. Compared with other species, fungi with antifungal activity usually contain expanded CAZyme families involved in fungal cell wall degradation, for example in Trichoderma atroviride and Trichoderma virens [14].

Secondary metabolites (SMs) are defined as bioactive, small molecules that are not essential to the growth of an organism [15]. Studies of SMs in Sarocladium genus have mainly focused on phytotoxins, as most species are plant pathogens. Two phytotoxins, helvolic acid and cerulenin, have been detected in liquid culture of S. oryzae, the pathogen causing rice sheath-rot, and were also found in infected rice sheath tissues $[16,17]$. Helvolic acid is a tetracyclic triterpenoid and can affect chlorophyll biosynthesis. Cerulenin is an epoxydodecadienamide that can inhibit polyketide and fatty acid synthesis by inhibiting malonyl-ACP:acylACP condensation [18]. The biosynthetic pathways of helvolic acid have been elucidated in Aspergillus flavus and Metarhizium anisophilae [19, 20]. When the S. oryzae genome was sequenced, Hittalmani et al. identified nine candidate genes involved in the helvolic acid biosynthesis pathway, based on protein homology analysis in S. oryzae [6].

Besides biosynthetic gene cluster (BGC) of helvolic acid, S. oryzae also contains other kinds of BGCs with unknown function, such as such as polyketide synthases (PKS), nonribosomal peptide synthetases (NRPS) and hybrids (PKSNRPS) [6]. NRPSs and PKSs are both large, multi-modular enzymes. NRPS modules contain three primary functional domains: Adenylation (A), thiolation (T), and condensation (C) [21]. PKS modules usually contain ketosynthase (KS), malonyl-CoA:acyl carrier protein transacylase (MAT), acyl carrier protein (ACP), ketoreductase (KR), and dehydratase (DH) [22]. Their high level of amino acid and nucleotide conservation mean that the A domains and KS domains are frequently used to reconstruct the evolutionary histories of NRPS and PKS, respectively [23, 24]. Backbone biosynthetic genes are often clustered with different kinds of enzyme-coding genes, such as cytochrome P450, methyltransferase, and hydroxylase. Usually, BGCs are highly distinct, and even between similar fungi whose genomes exhibit high sequence and synteny conservation, the identity and total number of BGCs can vary widely [25]. Currently, genomic studies are the best way to obtain a global view of fungal BGCs and comparative genomics allows the analysis of a fungus' potential to produce SMs [26].

In the present study, we report a gapless genome sequence of $S$. brachiariae, an endophytic fungus isolated from Brachiaria brizantha, which has the prospect of being applied as a biocontrol-agent. To understand the important pathways and genes utilized by $S$. brachiariae to carry out its antifungal and endophytic activities, we compare its genome with that of S. oryzae. After a general genome comparison, this study focused on comparing genes involved in CAZymes and SM biosynthesis. The results showed that $S$. brachiariae has more CAZymes involved in plant cell wall degradation and more CAZymes involved in fungi cell wall degradation, compared with those in $S$. ory$z a e$. Based on Antibiotics \& Secondary Metabolite Analysis Shell (antiSMASH) analysis, we identified a contact helvolic acid biosynthetic gene cluster (BGC) for the first time in $S$. oryzae. In addition, antiSMASH analysis result also indicated that S. brachiaria had seven fewer terpene gene 
clusters, including the helvolic acid BGC, compared with those in S. oryzae. Synteny analysis of PKS, NRPS, and PKS/NRPS gene clusters between $S$. brachiariae and $S$. oryzae revealed that only $37.5 \%$ of the tested clusters have good synteny, while $63.5 \%$ have no or poor synteny. This indicated that the $S$. brachiariae could potentially synthesize a variety of unknown-function secondary metabolites, which may play an important role in adaptation to its endophytic lifestyle and antifungal activity. The information contained in this study will be helpful to understand the biological nature of the endophytic fungus S. brachiariae and will widen our understanding of the Sarocladium genus.

\section{Results}

\section{General genome features and annotation}

The genome of S. brachiariae was sequenced to 191.0x coverage using a newly developed Single Molecule RealTime (SMRT) sequencing technique, which can assist in obtaining gapless eukaryotic genome sequences [27]. CANU was used for de novo assembly of the sequencing data $(6,115,460,666 \mathrm{bp}$ clean reads), which generated 19 contigs with a N50 length of $3.27 \mathrm{Mb}$ (Table 1). The genome assembly comprises approximately $31.86 \mathrm{Mb}$, which is slightly smaller than that of $S$. oryzae ( 32.40 Mb, GCA_001972265.1), and both of them are smaller than the average genome size of the Ascomycota (36.91 $\mathrm{Mb})[6,28]$. The completeness of the genome assembly was assessed using BUSCO (benchmarking universal single-copy Orthologs; https://busco.ezlab.org/), which showed that 1287 out of the 1375 (97.8\%) gene groups required for the correct assembly of Ascomycota are present in the $S$. brachiariae scaffolds assembly (Additional file 2: Table S1.1). Total RNA of S. brachiariae was extracted from mycelia collected from both PDA plates and PDB culture inoculated for 7 days. We got 93, 212,574 clean reads and 13,889,709,506 clean bases using Illumina novaseq 6000 system (Additional file 2: Table S1.2). TopHat2 analysis result indicated $89.4 \%$ genome pairs of $S$. brachiariae could be mapped with RNAseq result (Additional file 2: Table S1.3). Besides RNAseq, 22 genes from S. brachiariae were chosen for

Table 1 Genome characteristics and assembly features of S brachiariae and S. oryzae

\begin{tabular}{lll}
\hline Genome & $\begin{array}{l}\text { S. brachiariae } \\
\text { HND5 }\end{array}$ & $\begin{array}{l}\text { S. oryzae } \\
\text { IMl 501407 }\end{array}$ \\
\hline Total genome size (bp) & $31,862,451$ & $32,403,370$ \\
Number of contigs & 19 & 215 \\
Total assembly gap length & 0 & 64,323 \\
Depth of genome coverage (x) & 191 & 336 \\
N50 value (bp) & $3,269,626$ & 322,791 \\
G + C content (\%) & 52.04 & 53.1 \\
\hline
\end{tabular}

PCR validation of gene prediction result and all these genes could be amplified from genome DNA of S. brachiariae (Additional file 1: Figure S1; Additional file 8). These findings suggested that this assembly and in silico gene prediction is intact and robust.

Compared with S. oryzae (53.1\%), S. brachiariae has a lower GC content genome (52.04\%) (Table. 1).The $S$. brachiariae genome contains 55 rRNAs, 24 snRNAs, 1 miRNA, and 78 tRNAs (Additional file 2: Table S2). The de novo and reference based repeat analysis showed that $1.39 \%$ of genome is repetitive (Additional file 2: Table S3). A total 9903 protein-coding genes were predicted in the S. brachiariae genome, and $95.67 \%$ of predicted genes in S. brachiariae could be annotated. Among these predicted genes, 3801 (31.1\%), 5443 (54.96\%), 2999 (30.28\%), 9464 (95.57\%), and 6919 (69.87\%) could be annotated based on Clusters of Orthologous Groups (COG), gene ontology (GO), Encyclopedia of Genes and Genomes (KEGG), the non-redundant protein database (NR), and Swiss-Prot (Additional file 2: Table S4).

\section{Orthology analysis of S. brachiariae and another five Sordariomycetes fungi}

Orthology is a very efficient way to identify differences and similarities between model organisms and uncharacterized genomes [29]. An OrthoMCL analysis was performed on $S$. brachiariae and five other Sordariomycetes fungi $(\mathrm{Fu}$ sarium oxysporum, F. graminearum, S. oryzae, Acremonium chrysogenum, and Magnaporthe oryzae) with known genomes. A Venn diagram of the OrthoMCL analysis showed that $S$. brachiariae shares 5243 genes with the other five Sordariomycetes fungi (Fig. 1). The two Sarocladium species shared similarly low number of unique genes, S. brachiariae harbored the lowest number (101) of unique genes and $S$. oryzae harbored 119 unique genes in this group: F. oxysporum, F. graminearum, A. chrysogenum, and $M$. oryzae possessed more unique genes $(1553,134$, 140 , and 542 respectively).

\section{Phylogenetic relationship}

Based on Sarocladium phylogenetic relationship with other Sordariomycetes fungi $[2,3]$, seven Sordariomycetes genomes, including two endophytic fungi (Pochonia chlamydosporia and Coniochaeta ligniaria), five plant pathogens (Magnaporthe grisea, Verticillium dahliae, F. oxysporum, F. graminearum, and S. oryzae), and 1 marine fungus (A. chrysogenum), were used for phylogenomic analysis with S. brachiariae. Single-copy orthologous proteins were used to build the phylogenetic tree. The Maximum Likelihood phylogeny tree was generated by the RaxML [30] method based on the GTRGAMMA model. The result revealed that S. brachiariae was evolutionarily close to $S$. oryzae, a plant pathogen that causes sheath rot disease on rice (Fig. 2) [6]. In addition, $S$. brachiariae was also close to the other two 


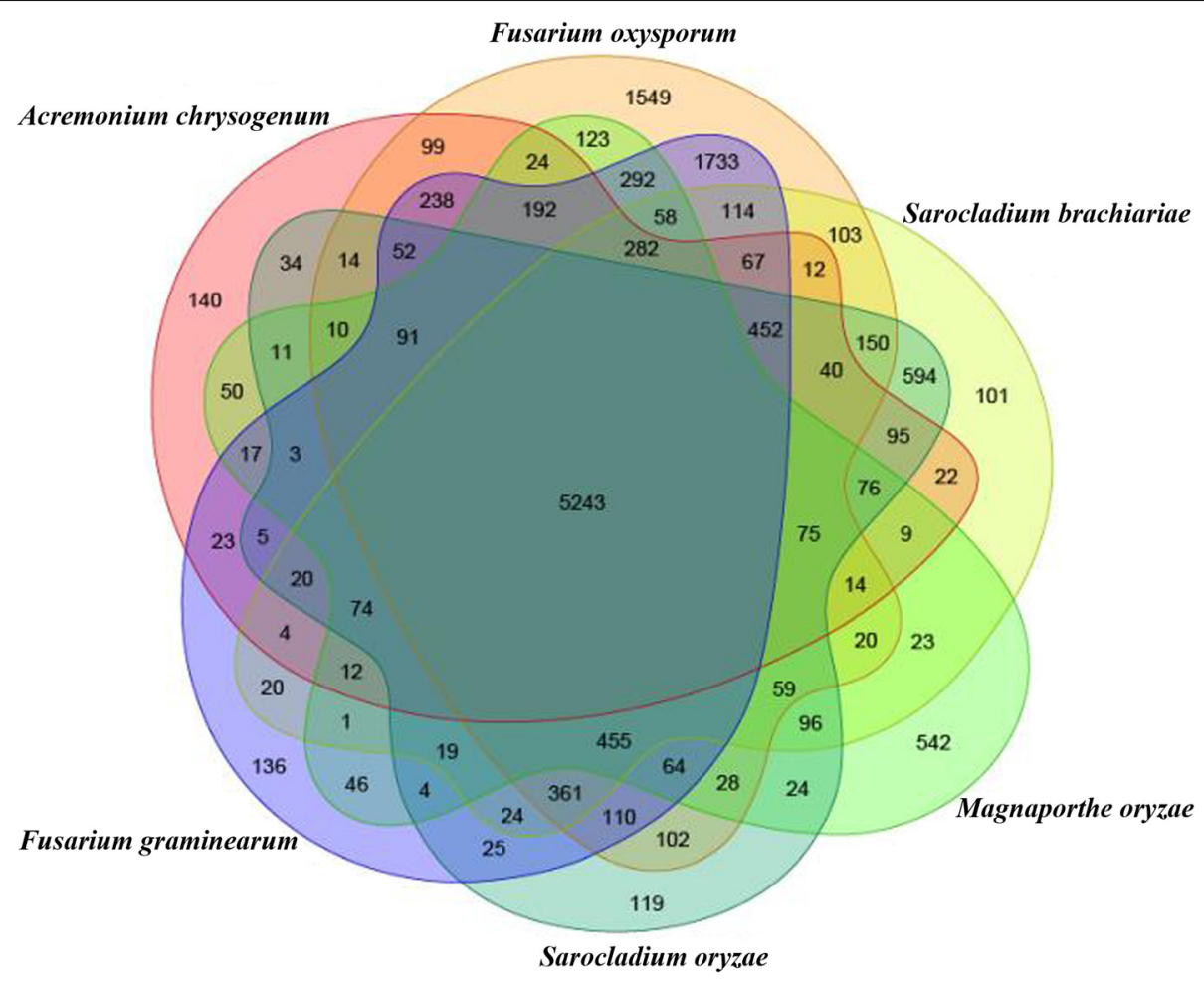

Fig. 1 Venn diagram showing shared and distinct orthologous gene families in Sordariomycetes fungi. The proteomes of Fusarium oxysporum, F. graminearum, S. oryzae, S. brachiariae, Acremonium chrysogenum, and Magnaporthe oryzae were clustered using orthoMCL. The numbers of genes in each species are shown in parenthesis

plant pathogens, Fusarium oxysporum and F. graminearum, and one endophytic fungus Pochonia chlamydosporia (Fig. 2).

\section{Functional annotation}

Using euKaryotic Orthologous Groups (KOG) [31] functional classification, we assigned functions to proteins based on sequence similarity (Fig. 3; The raw data are available in Additional file 2: Table S5). Of the total predicted proteins, $3466 \mathrm{~S}$. brachiariae proteins and $3516 \mathrm{~S}$. oryzae proteins had KOG hits. These were classified into four main groups: Function poorly characterized, Metabolism, Intracellular processes, and Information storage/ processing. KOG analyses revealed that the global pattern of protein allocation of $S$. brachiariae was very similar to that $S$. oryzae, indicating that these two strains are closely related and might have similar ecological niches. However, in one category, "Secondary metabolites biosynthesis, transport and catabolism", S. brachiariae had fewer hits (209) than S. oryzae (262), which indicated the secondary metabolites of these two species might be very different.

\section{Whole-genome synteny comparisons between $S$. brachiariae and S. oryzae}

The phylogenomic analysis revealed that $S$. brachiariae is evolutionarily close to $S$. oryzae; therefore, we performed synteny comparison between these two species. The MUMmer software was used to do the analysis and synteny dotplot was generated using mummerplot [32]. The generated synteny dot-plot showed the macrosynteny between the two genomes and high levels of sequence homology to each other with more than 95\% sequence identity (Fig. 4). Especially, contigs $0,1,2,4,5$, and 6 of S. brachiariae corresponded well with contigs $1,2,3,7,5$, and 10 of $S$. oryzae (Fig. 4). $25.81 \%$ of $S$. brachiariae genome and $24.49 \%$ of $S$. oryzae gnome shared high synteny, indicating these two Sarocladium species share conserved and core genes.

\section{Carbohydrate-active enzymes}

Carbohydrate-Active Enzymes (CAZymes) are crucial for fungal biological activity. For plant pathogens and endophytes, CAZymes are responsible for degradation of the host plant cell and to establish colonization. In addition, biocontrol fungi can use CAZymes to destroy the cell walls of pathogens and nematodes [10, 13]. Using the Carbohydrate Active Enzymes database, we annotated 1574 CAZyme proteins distributed across 155 CAZyme families in the S. brachiariae genome and 1551 CAZyme proteins distributed across 154 CAZyme families in S. oryzae genome. Among all CAZymes, 11.9\% (187) in S. brachiariae and $10.8 \%$ (167) in S. oryzae are secreted enzymes (Fig. 5; Additional file 2: Table S6). 


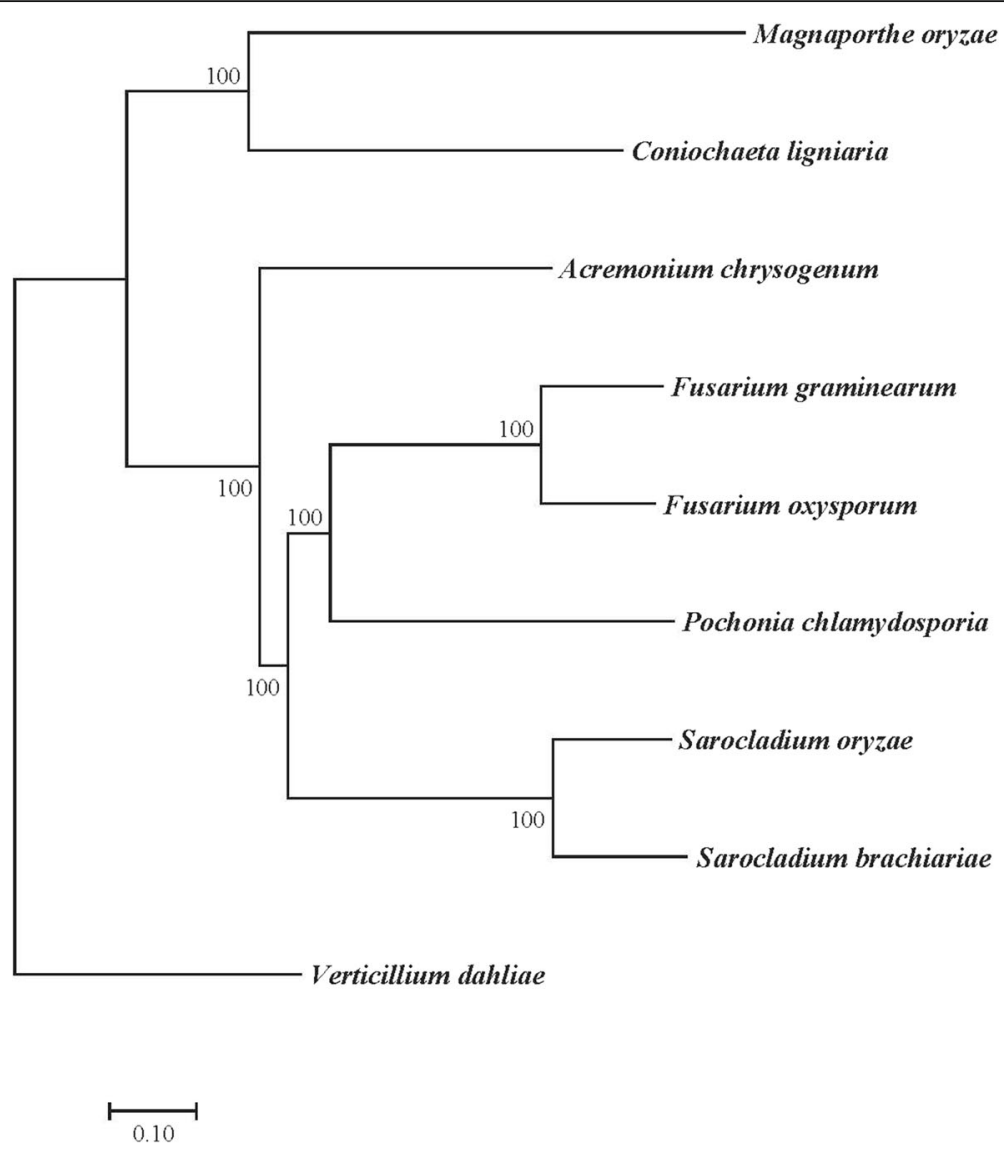

Fig. 2 Maximum Likelihood phylogenomic tree based on single copy orthologous genes

CAZymes were further classified by catalytic activity: Auxiliary activities (AAs), carbohydrate esterases (CEs), glycoside hydrolases (GHs), glycosyl transferases (GTs), and polysaccharide lyases (PLs). As shown in Fig. 5, CAZymes and secreted CAZymes from S. brachiariae and $S$. oryzae had a similar distribution. These two compared strains are plant associated fungi and S. brachiariae has antifungal activity; therefore, we analyzed the differences between CAZymes involved in plant and fungal cell wall degradation. According to the classification of Zhao [10] and Kubicek [8], CAZymes involved in plant cell wall degradation, such as cellulose, hemicellulose and pectin degradation, are listed in Table 2. CAZymes involved in chitin and $\beta-1,3-$ glucan degradation, which are major components of the fungal cell wall [13], are also listed in Table 3. The result of the comparison indicated that $S$. brachiariae possessed $14.9 \%$ more plant cell wall degradation CAZymes than $S$. oryzae. For the CAZymes involved in fungal cell wall degradation, S. brachiariae had 33.3\% more of secreted CAZymes than S. oryzae. This result indicated that $S$. brachiariae might have better fungal cell wall degradation ability than $S$. oryzae.

\section{Secondary metabolite gene clusters}

By definition, SMs are small bioactive molecules that were not strictly necessary for growth and development of an organism [25]. However, SMs are important for fungi's lifestyle, such as the antibiotics produced by biocontrol fungi and the phytotoxins synthesized by plant pathogens. The genes required for SM synthesis are usually arranged in a multigene biosynthetic gene cluster in fungi [33]. To analyze the SM synthesis potential of $S$. brachiariae, we used antiSMASH 3.0 (fungi view) to identify gene clusters in the genome of S. brachiariae. We also analyzed the genome of S. oryzae (GenBank: BCHE00000000.1) for comparison. The list of S. brachiariae and S. oryzae putative SM clusters and their genomic coordinates were shown in Additional file 2: Table S7.

As shown in Table 4, S. brachiariae has 34 SM gene clusters, including 7 non-ribosomal peptide synthase (NRPS) clusters, 12 polyketide synthase (PKS) clusters, 5 PKS/NRPS clusters, 6 terpene synthase clusters, and 4 other clusters. Compared with S. brachiariae, S. oryzae had 4 fewer PKS cluster, 6 more PKS/NRPS clusters, and 7 more terpene synthase clusters. 


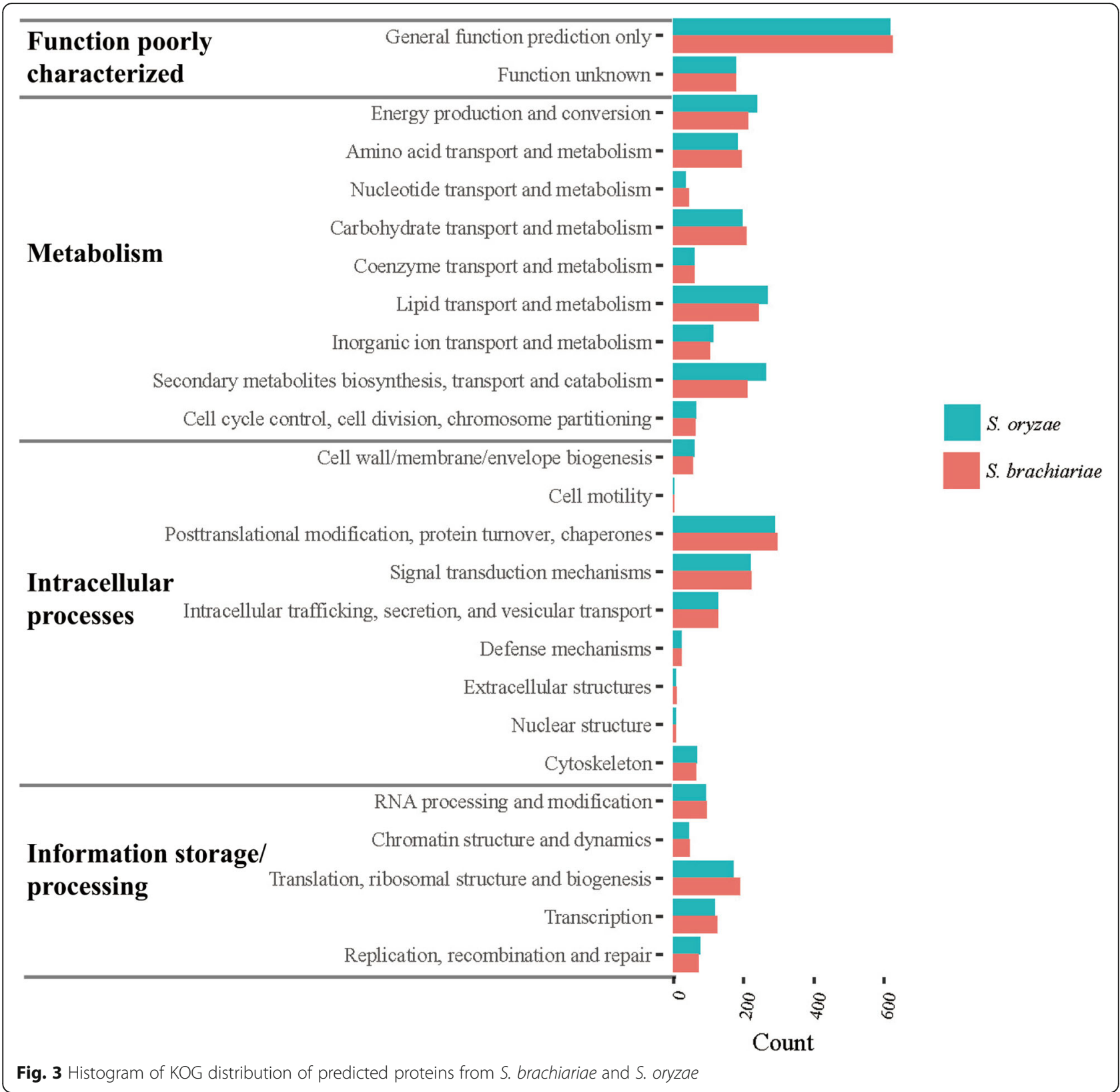

\section{Helvolic acid biosynthetic gene cluster analysis in}

\section{S. brachiariae and S. oryzae}

Helvolic acid and cerulenin were the two main phytotoxic metabolites synthesized and secreted by the rice pathogen S. oryzae [6, 34]. As an initial event in pathogenesis, helvolic acid and cerulenin can alter membrane permeability and cause electrolyte leakage [16]. The biosynthesis pathway for helvolic acid has been elucidated in Aspergillus fumigatus Af293 [19, 35]. There are nine genes in the helvolic acid synthesis cluster, including an oxidosqualene cyclase (OSC), a short-chain dehydrogenase/reductase (SDR), a 3-ketosteroid- $\Delta 1$-dehydrogenase (KSTD), two acyltransferases, and four cytochrome
P450s (CYP5081 family). Based on BLASTN search results, Hittalmani reported that nine genes are involved in helvolic acid biosynthesis in S. oryzae Saro-13 strain [6]. However, these nine genes spread across the whole genome and did not form a biosynthetic gene cluster (BGC). In the present study, using antiSMASH, we located a gene cluster in S. oryzae JCM 12450 strain that shared $77 \%$ similarity with the helvolic acid BGC reported in $A$. fumigatus Af293 (MIBiG BGC: BGC0000686). We further annotated genes in this cluster, and found this cluster had a similar organization to the helvolic acid BGC reported in $M$. anisophilae. However, the newly identified helvolic acid BGC only contained eight genes and was lacking one 


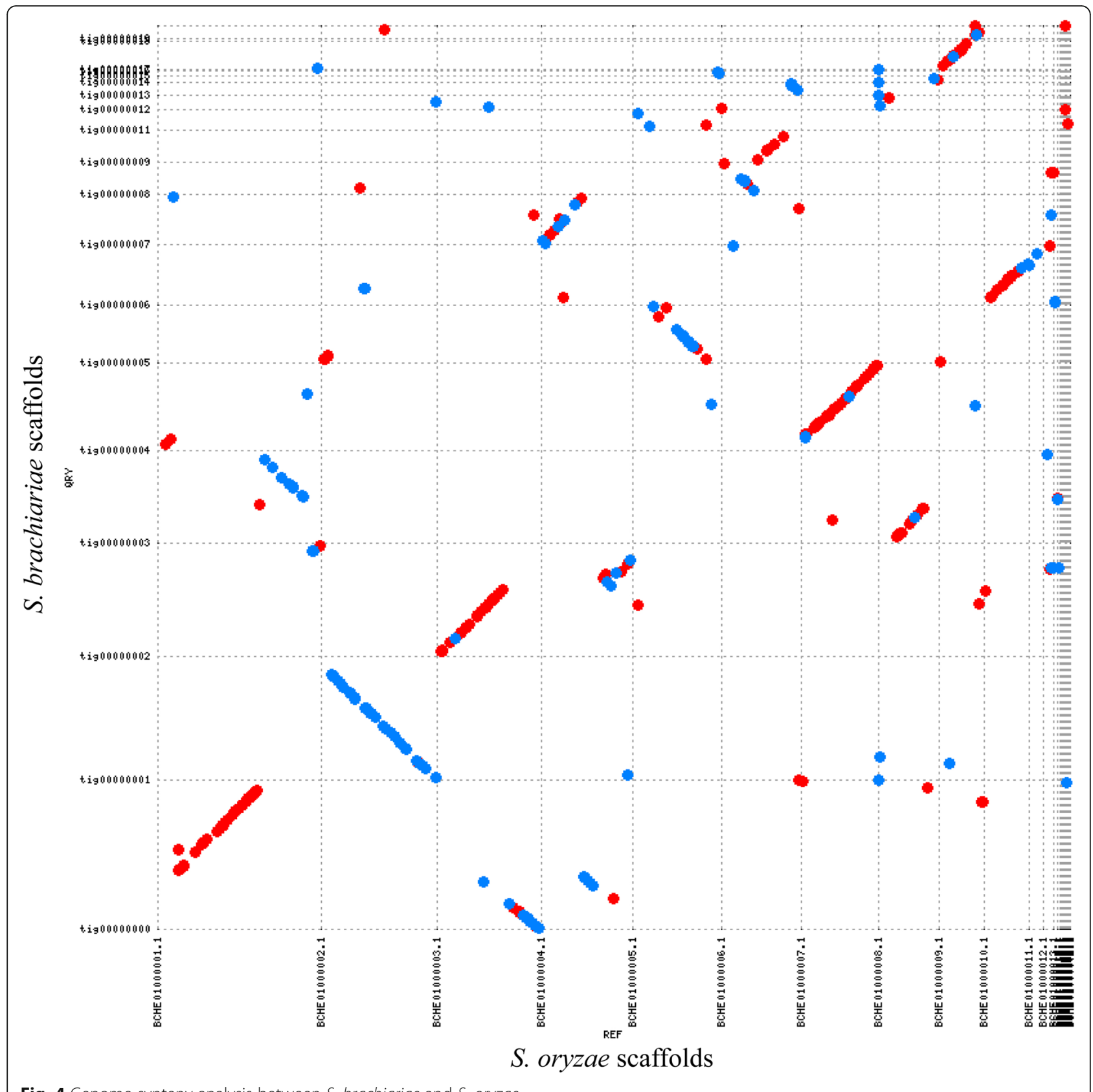

Fig. 4 Genome synteny analysis between S. brachiariae and S. oryzae

of the acyltransferase genes (Fig. 6, Additional file 3: Table S1). Therefore, this is a new type BGC for helvolic acid synthesis and is the first intact helvolic acid BGC identified in Sarocladium genus.

The antiSMASH result of $S$. brachiariae did not identify any cluster involved in helvolic acid synthesis. Among the genes responsible for helvolic acid biosynthesis, SDR, acyltransferase, and $\mathrm{P} 450$ genes are numerous in fungal genomes, while OSC and KSTD are rare. Therefore, we performed TBLASTN searches of the $S$. brachiariae genome against OSC and KSTD protein sequences
(Afu4g14770, Afu4g14850) to locate the probable helvolic acid synthesis cluster. One OSC (WHWLZ9448) and one KSTD (WHWLZ3787) encoding gene were found in $S$. brachiariae genome. Fourteen genes around these two genes (within a distance of $\geq 10 \mathrm{~Kb}$ ) were further annotated; however, no further genes related to helvolic acid synthesis were found (Additional file 3: Table S2). In addition, a genome wide analysis for cytochrome $\mathrm{P} 450$ genes showed that S. brachiariae has 388 CYP genes, but none of them belong to the CYP5081 family (Additional file 2: Table S8), 


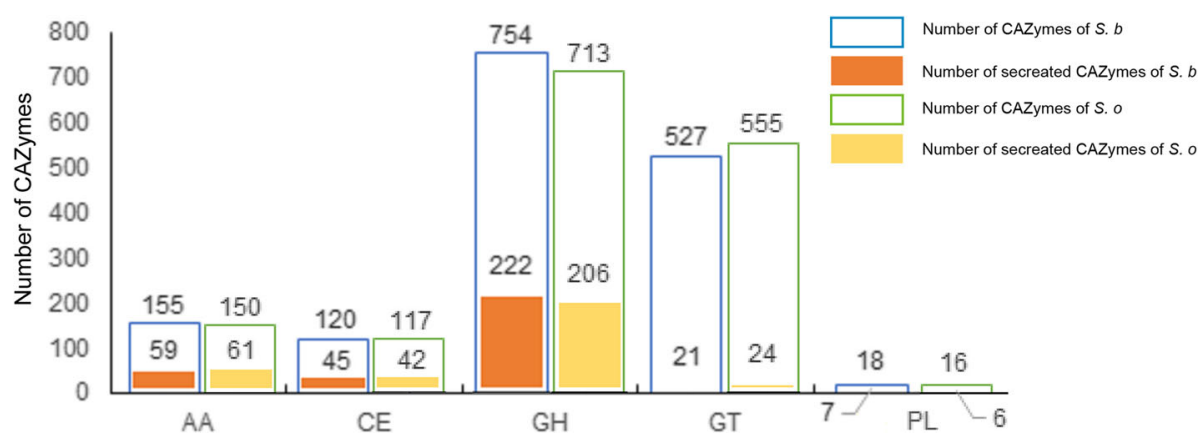

Fig. 5 Distribution of CAZymes and secreted CAZymes in S. brachiariae and S. oryzae

Table 2 CAZymes involved in plant cell wall degradation

\begin{tabular}{|c|c|c|c|c|c|}
\hline \multirow[t]{2}{*}{ Substrates } & \multirow[t]{2}{*}{ Family } & \multicolumn{2}{|l|}{ S. brachiariae } & \multicolumn{2}{|l|}{ S. oryzae } \\
\hline & & Copy number & Secreated copy number & Copy number & Secreated copy number \\
\hline \multirow[t]{8}{*}{ Cellulose } & $\mathrm{GH} 1$ & 4 & 1 & 5 & 1 \\
\hline & $\mathrm{GH} 3$ & 29 & 4 & 27 & 5 \\
\hline & GH5 & 20 & 8 & 23 & 9 \\
\hline & $\mathrm{GH} 12$ & 14 & 3 & 8 & 3 \\
\hline & $\mathrm{GH} 45$ & 1 & 1 & 2 & 2 \\
\hline & $\mathrm{GH} 6$ & 4 & 3 & 4 & 2 \\
\hline & $\mathrm{GH7}$ & 7 & 7 & 7 & 7 \\
\hline & GH74 & 1 & 1 & 1 & 1 \\
\hline \multirow[t]{16}{*}{ hemicellulose } & GH10 & 5 & 3 & 6 & 2 \\
\hline & GH11 & 10 & 9 & 10 & 9 \\
\hline & GH3O & 11 & 3 & 9 & 1 \\
\hline & $\mathrm{GH} 115$ & 2 & 0 & 1 & 0 \\
\hline & $\mathrm{GH} 27$ & 6 & 3 & 6 & 2 \\
\hline & $\mathrm{GH} 29$ & 0 & 2 & 4 & 2 \\
\hline & GH35 & 3 & 1 & 3 & 1 \\
\hline & GH36 & 16 & 0 & 13 & 0 \\
\hline & GH39 & 0 & 2 & 7 & 3 \\
\hline & GH51 & 2 & 0 & 2 & 0 \\
\hline & GH53 & 2 & 2 & 1 & 1 \\
\hline & GH54 & 6 & 4 & 3 & 3 \\
\hline & GH62 & 3 & 3 & 1 & 1 \\
\hline & GH67 & 2 & 1 & 2 & 1 \\
\hline & GH93 & 6 & 1 & 6 & 1 \\
\hline & $\mathrm{GH} 43$ & 44 & 12 & 45 & 9 \\
\hline \multirow[t]{7}{*}{ Pectin } & $\mathrm{GH} 105$ & 2 & 1 & 4 & 1 \\
\hline & $\mathrm{GH} 28$ & 16 & 3 & 16 & 2 \\
\hline & GH78 & 10 & 0 & 6 & 0 \\
\hline & GH88 & 2 & 0 & 2 & 0 \\
\hline & PL1 & 6 & 4 & 6 & 3 \\
\hline & PL3 & 2 & 2 & 2 & 2 \\
\hline & PL4 & 7 & 1 & 5 & 0 \\
\hline Total number & & 243 & 85 & 237 & 74 \\
\hline
\end{tabular}


Table 3 CAZymes involved in fungal cell wall degradation

\begin{tabular}{|c|c|c|c|c|c|}
\hline \multirow[t]{2}{*}{ Substrates } & \multirow[t]{2}{*}{ Family } & \multicolumn{2}{|l|}{ S. brachiariae } & \multicolumn{2}{|l|}{ S. oryzae } \\
\hline & & Copy number & Secreated copy number & Copy number & Secreated copy number \\
\hline \multirow[t]{4}{*}{ Chitin } & $\mathrm{GH} 18$ & 132 & 17 & 127 & 13 \\
\hline & $\mathrm{GH} 2 \mathrm{O}$ & 9 & 0 & 8 & 0 \\
\hline & GH75 & 4 & 1 & 4 & 1 \\
\hline & GH76 & 20 & 0 & 20 & 0 \\
\hline \multirow[t]{6}{*}{$\beta$-1,3-glucan } & $\mathrm{GH} 17$ & 21 & 1 & 21 & 2 \\
\hline & GH55 & 11 & 1 & 11 & 2 \\
\hline & $\mathrm{GH} 71$ & 4 & 0 & 5 & 0 \\
\hline & $\mathrm{GH} 72$ & 8 & 0 & 8 & 0 \\
\hline & GH81 & 3 & 0 & 2 & 1 \\
\hline & GH16 & 65 & 16 & 47 & 8 \\
\hline Total number & & 277 & 36 & 253 & 27 \\
\hline
\end{tabular}

Abbreviations: $\mathrm{GH}$, glycoside hydrolase

which is critical for helvolic acid synthesis [20]. Taken together, these results strongly suggest that $S$. brachiariae lacks the genome resources to synthesize helvolic acid. S. brachiariae is an endophyte and can coexist with host plants without causing any damage [4]. Therefore, the lack of the phytotoxin helvolic acid BGC in S. brachiariae might be an adaptation method for its endophytic lifestyle.

\section{Phylogenomic analysis of NRPS, PKS, and PKS/NRPS genes of $S$. brachiariae and S. oryzae}

Many fungal SMs are synthesized by multimodular enzymes, NRPSs, PKSs, or hybrid PKS/NRPS. To determine differences between the secondary metabolomes of $S$. brachiariae and S. oryzae, we analyzed the phylogenomic relationships of NRPSs, PKSs, and PKS/NRPS identified in these two species. As A domain and KS domain are relatively conserved in NRPS and PKS [23, 36]; therefore, the phylogenetic relationships among NRPSs, PKSs, and PKS/NRPS were analyzed based on the A domain or KS domain sequences. Phylogenetic analysis of the A domains revealed that NRPSs from S. brachiariae and $S$. oryzae could be grouped into six clades, NRPS1-

Table 4 The secondary metabolites gene clusters in S. brachiariae and S. oryzae

\begin{tabular}{lll}
\hline Genome & S. brachiariae & S. oryzae \\
\hline NRPS & 7 & 7 \\
PKS & 11 & 8 \\
PKS-NRPS & 5 & 10 \\
Terpene & 6 & 13 \\
Other & 5 & 8 \\
Total & 34 & 46
\end{tabular}

Abbreviations: NRPS, nonribosomal peptides synthase; PKS, polyketide synthase; PKS-NRPS: hybrid NRPS-PKS enzymes
3 clades are mainly mono-/bimodular NRPSs and NRPS 4-6 clades are multimodular NRPSs (Fig. 7). The KS domain phylogenetic analysis indicated that the PKSs from the two compared strains could be grouped into five clades (Fig. 8). PKS/NRPS contain both A and KS domains; the PKS/NRPS phylogenetic relationships were analyzed using the A and KS domains, separately. As shown in Figs. 7 and 8, PKS/NRPSs from the two compared strains formed two clades and A domain and KS domain phylogenetic analysis showed the same result. Both clades had more than $50 \%$ bootstrap support. Further analysis of the domain structure revealed that synthases from the same clade shared a similar domain structure (Figs. 7 and 8). Most clades contain an equal number synthases from $S$. brachiariae and $S$. oryzae, such as NRPS clades 1, 3, 4 and 5; and PKS clade 1, 2, and 3. However, some clades contain unequal number of synthases, such as PKS clade 4, which contains one PKSs from $S$. oryzae and four PKSs from S. brachiariae. This indicated that high level gene duplication had happened during the evolution of SM-related genes in the Sarocladium genus.

\section{Synteny analysis of SM gene clusters of S. brachiariae and} S. oryzae

Except for backbone synthesis genes whose enzymatic products produce a core metabolite, such as NRPSs and PKSs, a contact biosynthetic gene cluster (BGC) also contains genes involved in product modification, transport, and transcription regulation [15]. Closely related species may share some specific BGCs, but the identity and total number of BGCs can vary widely between them [25]. To analyze the differences between BGCs from S. brachiariae and $S$. oryzae, synteny of BGCs in which the backbone genes are in the same clade were analyzed. Figures 9, 10, 11 showed that 42 of $48(87.5 \%)$ tested PKSs, NRPSs, and 


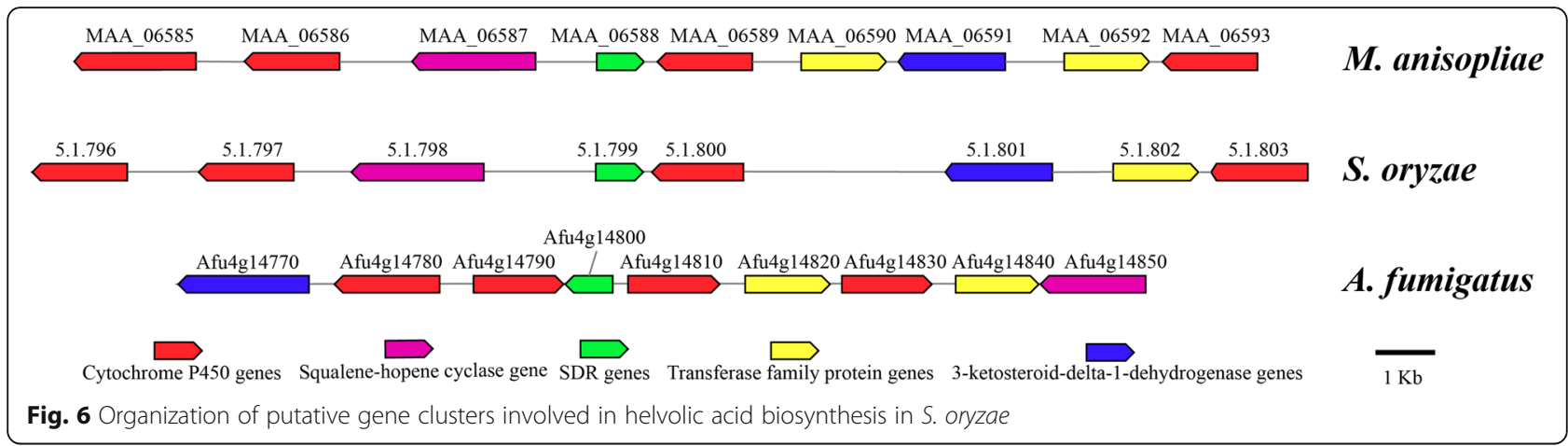

PKS/NRPSs gene clusters had some synteny between $S$. brachiariae and $S$. oryzae, and the remaining six clusters (12.5\%) did not have any synteny. Among the gene clusters with synteny, we found six pairs of clusters with the same gene content (S. brachiariae Cluster31-S. oryzae Cluster 33, S. brachiariae Cluster 7-S. oryzae Cluster 42, S. brachiariae Cluster 24-S. oryzae Cluster 44, S. brachiariae Cluster 29-S. oryzae Cluster 12, S. brachiariae Cluster 32-S. oryzae Cluster 26, and S. brachiariae Cluster $16-S$. oryzae Cluster 4 ), just $25 \%$ (12 of 48 ) of all the analyzed clusters. Three pairs of clusters (S. brachiariae Cluster 5-S. oryzae Cluster 12, S. brachiariae Cluster 6-S. oryzae Cluster 11, and S. brachiariae Cluster 33-S. oryzae Cluster 1) had basically the same gene content except for one or two genes, and accounted 12.5\% (6 of 48) of all the analyzed clusters. The remaining clusters with synteny had similar core genes but totally different contents of other genes, which accounted for $50 \%$ of the tested clusters. Taken together, $37.5 \%$ of PKSs, NRPSs, and PKS/NRPSs gene clusters between S. brachiariae and $S$. oryzae had good synteny, while most of these clusters, $62.5 \%$, had poor or no synteny. This result suggested a big difference between the secondary metabolisms of S. brachiariae and S. oryzae. This data also indicated that $S$. brachiariae could potentially synthesize a variety of unknown-function SMs, which may play an important role in adaptation to its endophytic lifestyle and antifungal activity.

\section{Discussion}

The Sarocladium genus presently encompasses 17 species, including three phytopathogens, two endophytes, six opportunistic human pathogens, and one mycoparasite [2, 3]. Studies on Sarocladium have been focused on plant and human pathogenic species because of their danger to crops and humans. To date, only three species have genome sequence data and all of them cause plant or human diseases. Among them, S. oryzae is the phytopathogenic species whose whole genome has been sequenced and published [6]. The other two species are human pathogens; the genome sequence of $S$. strictum has been determined, but no paper has been published (GCA_900290465.1) and the S. kiliense data comprises only raw sequence reads [37]. In addition to pathogenic species, endophytic Sarocladium species have been found in different kinds of plants, such as Brachiaria brizantha, maize, and water mint [4, 38, 39]. Two endophytic species, S. brachiariae and S. zeae, have demonstrated substantial biocontrol potential on phytopathogens [40]. However, the lack of a determined genome sequence has restrained further research on the life cycle and biocontrol mechanism of endophytic species. In the present study, we focused on deciphering the genomic basis underlying the differences between endophytic and pathogenic Sarocladium species. To this end, we generated a reference genome for the endophytic species, S. brachiariae, and performed a comparative analysis of genomic data between S. brachiariae and the phytopathogen, S. oryzae.

In our former result, we established the new Sarocladium species, Sarocladium brachiariae, based on morphological characteristics together with large subunit (LSU) and ITS rDNA sequences [4]. In the present study, we firstly analyzed the phylogenic relationship of S. brachiariae with eight fungal species that have close relationships with Sarocladium according to Summerbell et al [3]. The Maximum Likelihood phylogeny tree generated based on single-copy orthologous proteins supports our former result and S. brachiariae was classified with S. oryzae with $100 \%$ bootstrap support. The phylogenomic analysis presented in this study is also largely in agreement with typical phylogenetic studies that sampled species of Acremonium, Sarocladium, and other genera belonging to the Hypocreales and generated phylogenic relationships from Maximum Likelihood analysis via RAxML VI-HPC of ribosomal RNA gene (LSU + SSU) datasets $[2,3]$.

Plant cell walls are the first and major barriers to infection by fungal pathogens, and are also the main component of plant biomass. To penetrate into plant cells or use plant cells as a carbon source, plant parasitic fungi secrete a cocktail of hydrolytic enzymes known as carbohydrateactive enzymes (CAZymes) [41]. Zhao et al. identified and compared the full repertoires of CAZymes from symbiotic, biotrophic, hemibiotrophic, necrotrophic, and 


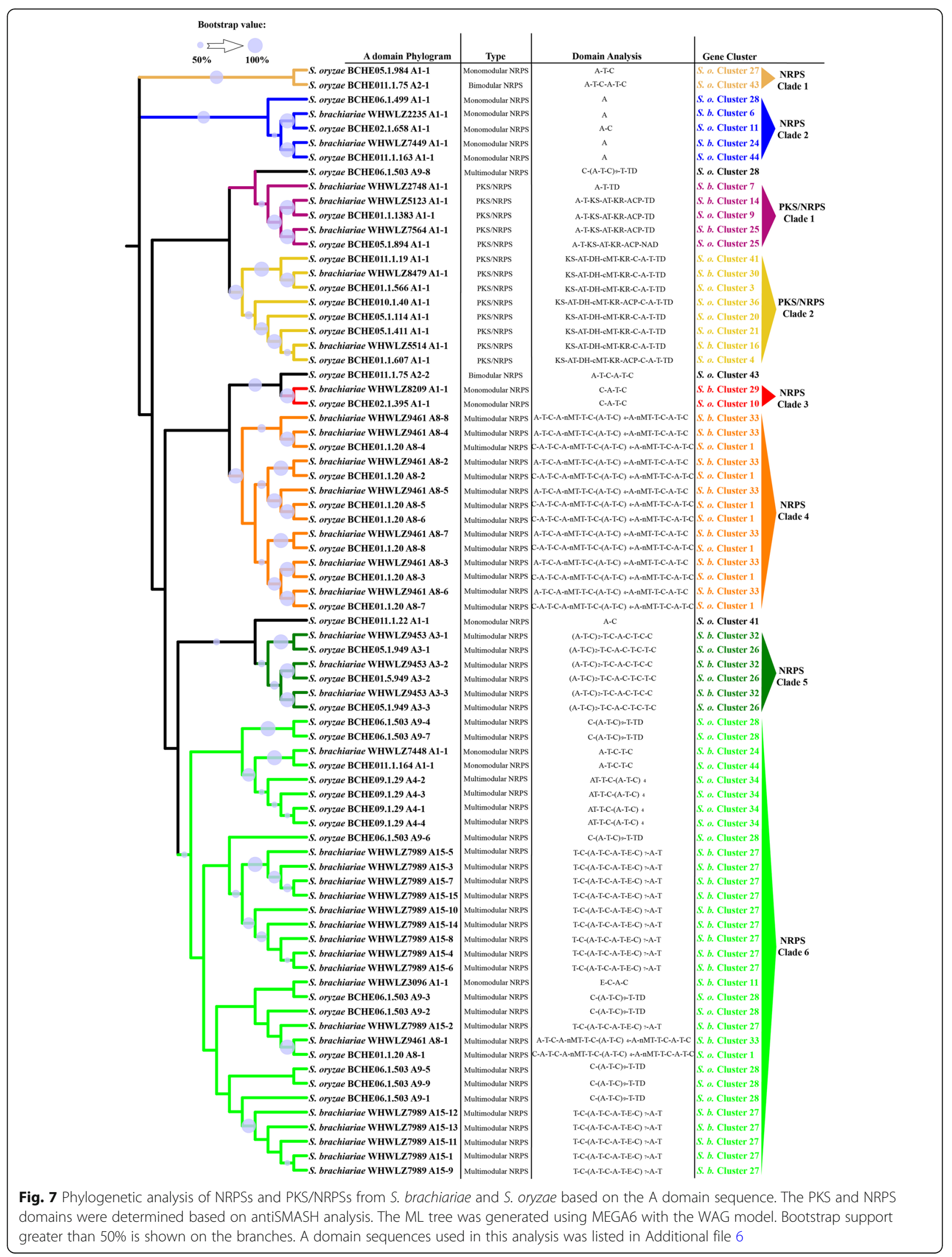




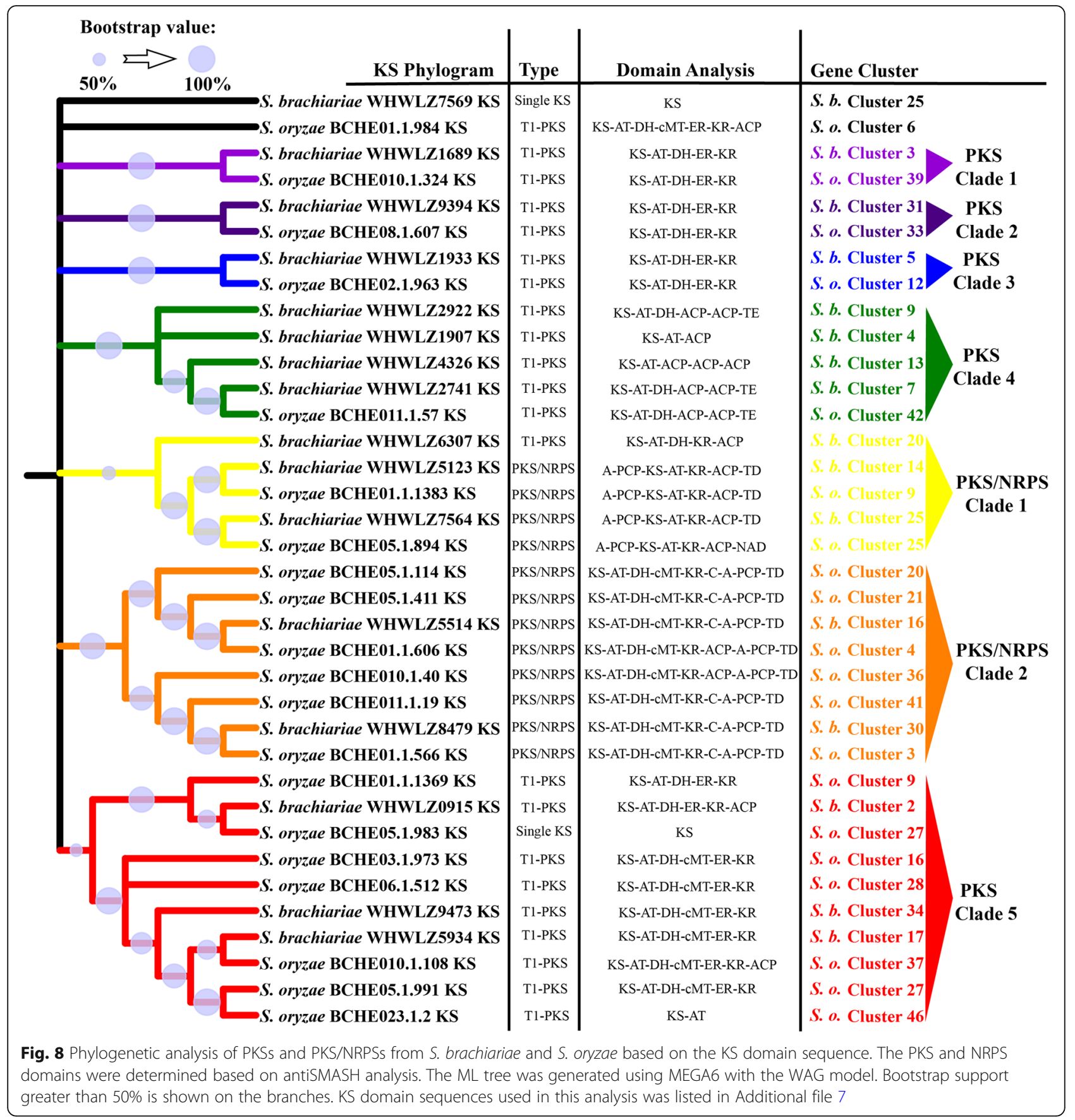

saprophytic fungi, and performed a comprehensive comparison of the distribution and abundance of CAZyme families. Based on their results, symbiotic and biotrophic fungi contain less CAZymes and less plant cell wall degrading enzymes than necrotrophic and hemibiotrophic fungi, such as GH61, GH78, PL1, and PL3. Most obviously, symbiotic and biotrophic fungi lack GH6 enzymes, which have endoglucanase and cellobiohydrolase activities [10]. In the present study, we identified that the endophytic fungus $S$. brachiariae has more CAZymes and plant cell wall degradation-related CAZymes than the phytopathogen S. oryzae.

In addition, four GH6 enzymes were identified in S. brachiariae. Recently, four endophytic fungi (Hypoxylon sp. CI4A, Hypoxylon sp. EC38, Hypoxylon sp. CO27 and Daldinia eschscholzii EC12) were reported to be rich in CAZymes and could deconstruct plant cell walls to produce mycodiesel. These four strains all harbor two GH6 enzymes [42]. Pochonia chlamydosporia is a soil fungus with a multitrophic lifestyle combining endophytic and 


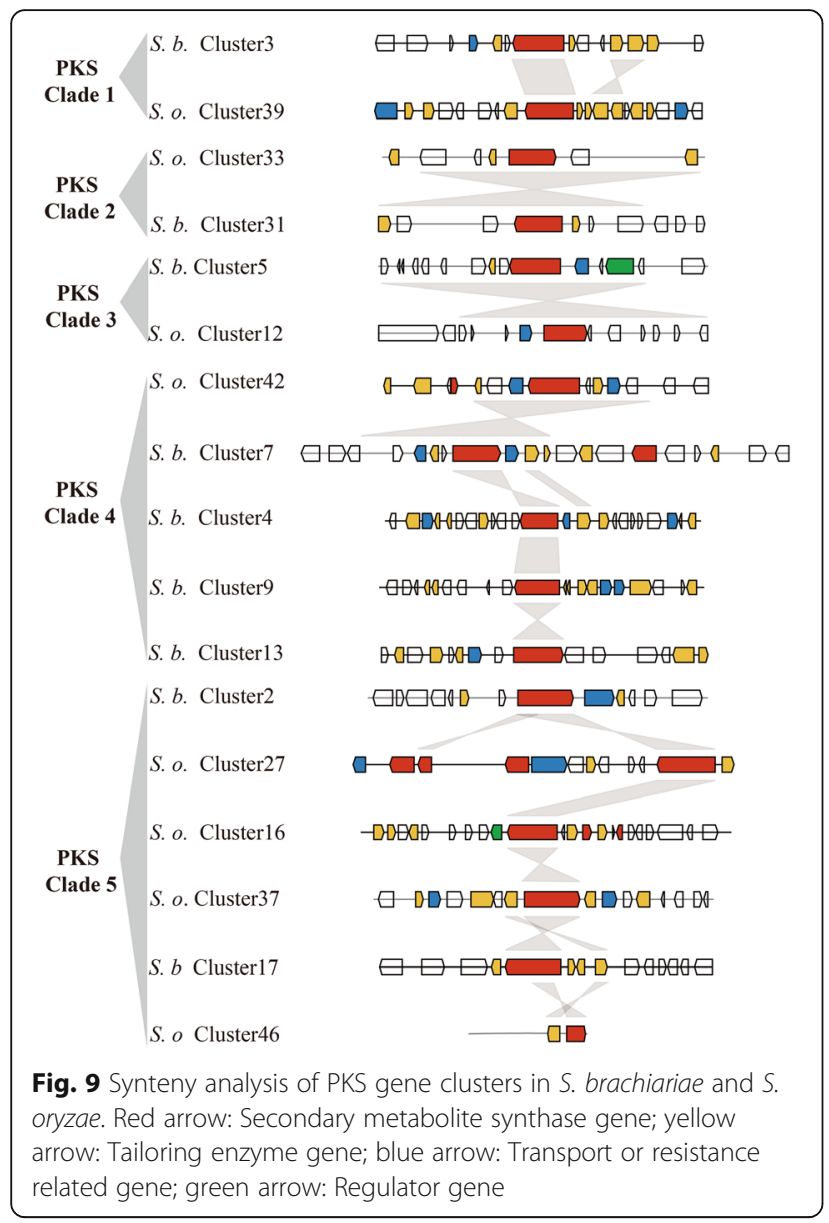

saprophytic behavior. Aranda-Martinez et al. showed that this strain also contains significant amounts of CAZymes involved in plant cell wall degradation [43]. On the basis of these results, we would like to propose the following two explanations: (1) endophytic fungi need a certain amount of cocktail of CAZymes to destroy plant cell wall before colonizing host plants or using plant cell wall polysaccharides as nutrients; (2) S. brachiariae might have a multitrophic lifestyle and turn to necrotrophic or hemibiotrophic under some circumstances [44-47].

Fungal cell walls, mainly composed of chitin and $\beta-(1$, 3)-glucan, are also an important barrier to protect fungi against invasion by mycoparasitic fungi or bacteria. Lysis of the cell wall is one of the key steps during mycoparasitism, and mycoparasitic fungi usually poss an expanded set of fungal cell wall degradation-related CAZymes, such as GH18 and GH75 [14, 17]. Trichoderma species are mycoparasitic fungi and are used as biocontrol agents against plant pathogenetic fungi. Trichoderma have an expanded repertoire of chitinases (GH18), chitosanases (GH75), and $\beta$-(1,3)-glucanases (GH55). In the present study, we also noted that $S$. brachiariae possess 33.3\% more secreted fungal cell wall degradation-related CAZymes than $S$. oryzae, especially chitinases (GH18) and $\beta$-(1,3)-glucanases (GH55, GH16) (Table 3). This result could partially explain the antifungal activity of S. brachiariae.

Phytotoxins are low-molecular-weight secondary metabolites capable of disturbing the vital activity of plant cells or causing their death at concentrations below 10 $\mathrm{mM}$. Phytopathogenic fungi are best known as phytotoxin producers [48]. Rice pathogenic $S$. oryzae produces

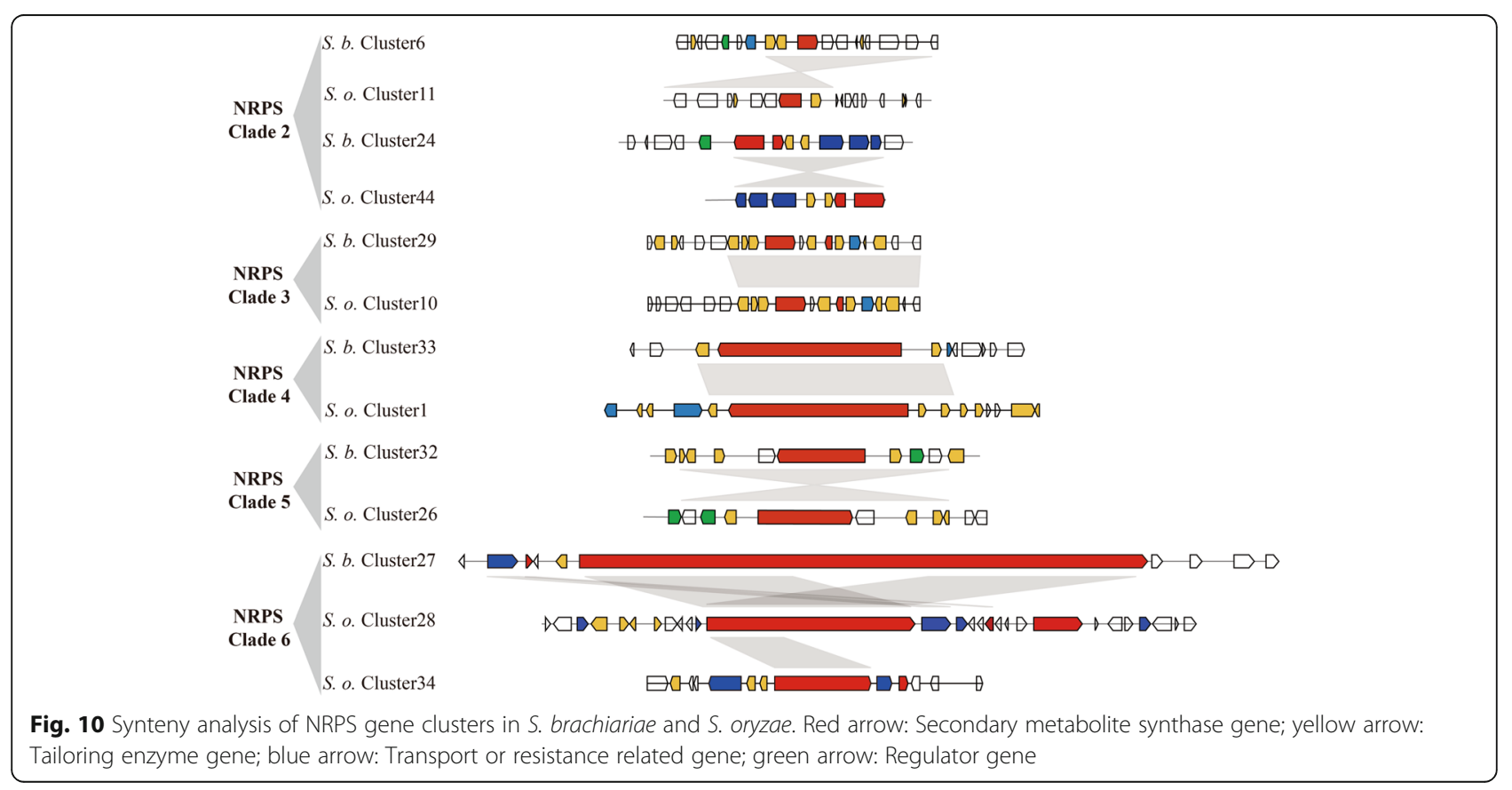




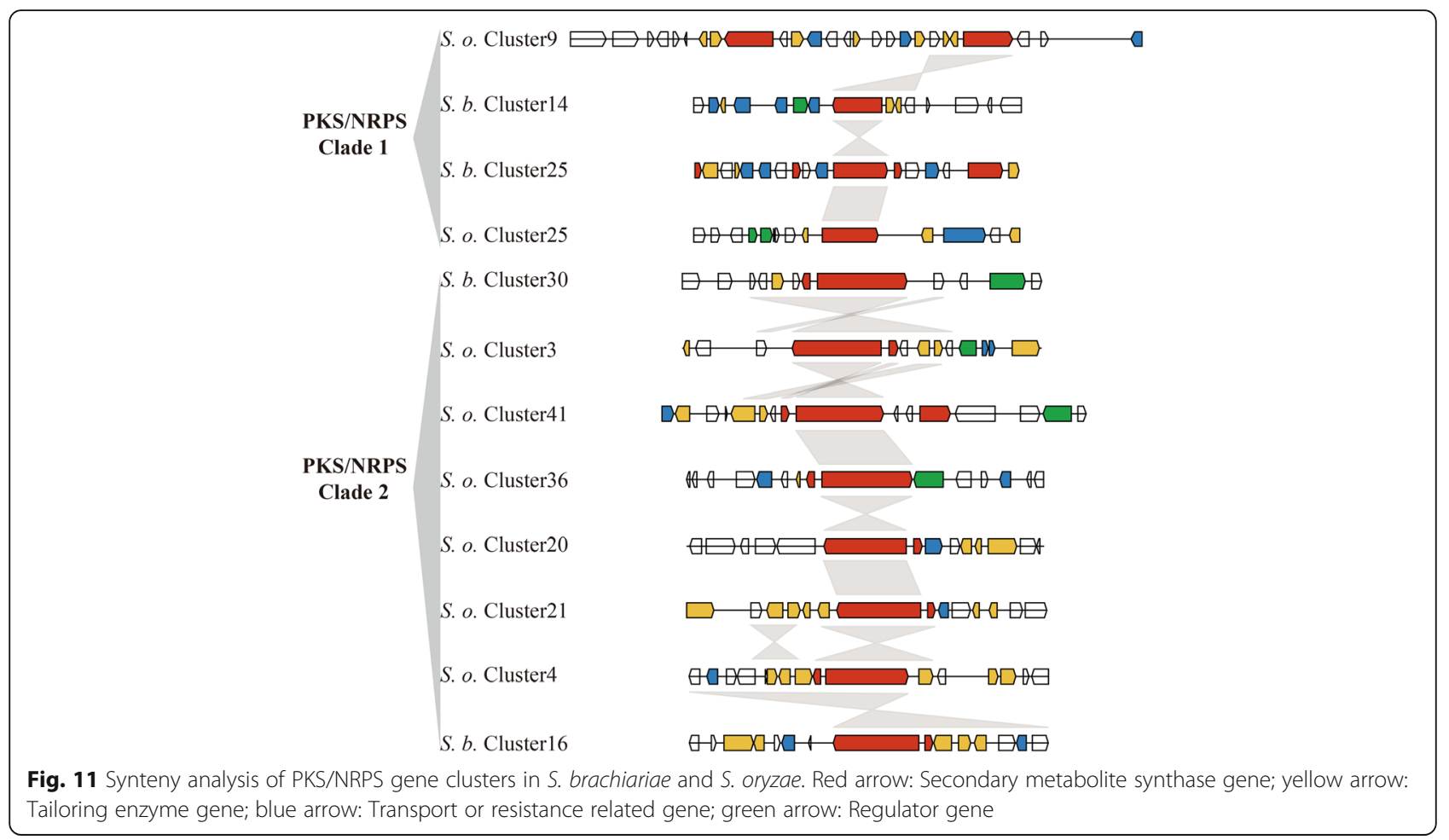

the phytotoxins helvolic acid and cerulenin, both of which cause necrosis and mimic the disease symptoms of sheath rot caused by $S$. oryzae [16, 49]. S. oryzae uses these two phytotoxins to change cell permeability, leading to leakage of electrolytes before invading the host tissue $[18,34]$. Cerulenin was discovered in the 1960 s as antibiotic because it could inhibit fatty acid synthesis in fungi; however, its biosynthesis process remains unclear [50]. Helvolic acid is synthesized by different fungi and its biosynthetic gene cluster (BGC) has been determined in A. fumigatus and M. anisophilae. The BGC responsible for helvolic acid synthesis contains one oxidosqualene cyclase, one 3 -ketosteroid- $\Delta 1$-dehydrogenase, one short-chain dehydrogenase/reductase, two acyltransferases, and four CYP5081 family cytochrome P450 genes $[19,20]$. Based on de novo sequencing data, Hittalmani et al. identified nine genes related to helvolic acid biosynthesis in $S$. oryzae Saro-13 strain using BLASTP searches of the $S$. oryzae proteome against $A$. fumigatus protein sequences [6]. These nine genes were spread across the whole genome and did not form a common BGC; however, this might have been caused by the poor assembly result (5856 contigs). Based on the antiSMASH analysis, we identified the BGC responsible for helvolic acid synthesis in $S$. oryzae on assembly GCA_001972265.1. The analysis identified that eight continuous genes form the BGC for helvolic acid in $S$. oryzae. Compared with the known BGCs in A. fumigatus and $M$. anisophilae, the newly found helvolic acid BGC contains one fewer acyltransferase gene. The structural arrangement of the $S$. oryzae BGC is more similar to that of $M$. anisophilae than to that of A. fumigatus. Lv et al. determined the function of each gene and the encoded protein reaction sequences by reconstitution of the nine genes from the BGC of $A$. fumigatus one by one in $A$. oryzae NSAR1. In their study, they proposed that the two acyltransferase had different active site, Held1 (the first acyltransferase) accounts for the acetylation of C-6 hydroxyl group, and Held2 (the second acyltransferase) acts on the C-12 $\beta$ hydroxyl group [35]. However, BGC in $S$. oryzae just has one acyltransferase gene that shares 69\% homologous with the Held1 gene. Therefore, we propose that the biosynthetic process of helvolic acid in S. oryzae is a little different to the known pathway and the newly identified acyltransferase could act on both C-6 and C-12 $\beta$ hydroxyl groups.

Gene loss is a widespread source of genetic variation that can cause adaptive phenotypic diversity [51]. In plant pathogenic and symbiotic fungi, losses of genes encoding CAZymes, secondary metabolite enzymes, and enzymes in the nitrogen and sulfur assimilation pathways enable biotrophs to adapt to plant hosts by avoiding recognition by the plant defense systems [52]. The best-known gene loss in phytopathogens is the absence of avirulence genes. Loss of avirulence genes could disable the resistant genes in the host plants and enable avirulent isolates to become virulent to phytopathogens, such as AVR-Pita in M. oryzae, AvrLm1 in Leptosphaeria 
maculans and NIP1 in Rhynchosporium secalis [53-55]. Using antiSMASH, we identified 44 BGCs in S. oryzae, including those encoding NRPSs, PKSs, PKS/NRPS hybrid, terpenes, and others. Meanwhile, in S. brachiariae, we found only 34 BGCs, 10 fewer than in S. oryzae. KOG distribution analysis also gave the same result, $S$. brachiariae had 53 fewer proteins belonging to "Secondary metabolites biosynthesis, transport and catabolism" than $S$. oryzae. These two strains share the same number of NRPSs; however, although numbers of the PKSs and PKS/NRPS hybrid are very different, the total number of PKSs and PKS/NRPS hybrid is similar. The biggest difference between the BGCs of these two Sarocladium sp. is the number of BGCs for terpenes. S. brachiariae (seven terpene BGCs) has $46 \%$ fewer than S. oryzae (13 terpene BGCs). Interestingly, the helvolic acid BGC is included in these six lost terpene BGCs. We further performed manual BLASTP searches of the S. brachiariae proteome against helvolic acid synthesis related proteins in S. oryzae. Based on the BLASTP result, there is one oxidosqualene cyclase and one 3-ketosteroid- $\Delta 1$-dehydrogenase encoding gene in the $S$. brachiariae genome; however, these two genes are located far from each other. In addition, we did not find any gene encoding a cytochrome P450 of the CYP5081 family in S. brachiariae. Therefore, the endophytic fungus S. brachiariae does not possess the genetic resources to synthesize helvolic acid. Helvolic acid is the major phytotoxin synthesized by $S$. oryzae; therefore, we speculated the lack of helvolic acid-related biosynthetic genes are the adaptive for $S$. brachiariae to form a mutualistic relationship with the host plant as an endophyte.

In plant-associated fungi, non-ribosomal peptides and polyketides have different functions, such as phytotoxins, mycotoxins, and antibiotics. These two different kinds of secondary metabolites are separately synthesized by NRPSs and PKSs, which are both large and multimodular enzymes [56]. The occurrence of horizontal gene transfer, gene duplication and loss, domain acquisition, and point mutations, mean that NRPS and PKS genes are generally recognized as rapidly evolving gene classes in fungi. Fast evolution leads to few clearly identifiable orthologs between species and highly discontinuous distributions, even between closely related species [23, 24]. However, within these two fast evolving enzymes, the ketosynthase (KS) domain and adenylation (A) domain have relatively conserved amino acid sequence in PKSs and NRPSs, respectively. Based on sequences of these two domains, Kroken et al. and Bushley et al. analyzed the phylogenomic relationships of known PKSs and NRPSs, separately [23, 36]. A few studies on secondary metabolites of Sarocladium sp. have focused on terpene phytotoxins, but none have investigated NRPSs and PKSs in Sarocladium sp. [6, 17, 34]. Based on the
Maximum Likelihood phylogenetic trees generated from the amino acid sequences of KS or A domains in S. brachiariae and S. oryzae, we found that PKSs, NRPSs, and PKS/NRPS with similar domain structures could be clustered together, with high bootstrap support (Figs. 7 and 8). This indicated that the two compared species share a close evolutionary relationship. In addition, we found a high level of gene duplication, especially in PKS Clade 4 (one PKS from S. oryzae, four PKSs from S. brachiariae) and PKS/NRPS Clade 2 (two PKS/NRPSs from S. brachiariae, six PKS/NRPSs from S. oryzae) (Figs. 7 and 8). Carbone et al. reported secondary metabolite biosynthetic gene duplication in Aspergillus, in which many copies of aflatoxin biosynthetic genes were found in the genome. They suggested the gene duplication drives the evolution of gene clusters involved in secondary metabolites synthesis [57]. This may have resulted the differences in BGCs between S. brachiariae and S. oryzae.

However, core genes are not sufficient to synthesize secondary products, they need the help of other genes around them, which constitute a contact biosynthetic gene cluster (BGC). Based on their phylogenetic relationship, we analyzed the syntenic relationships of BGCs in which core genes were clustered into one clade. The results indicated that just $37.5 \%$ of tested clusters had good synteny, $50 \%$ only shared similar core genes, and $12.5 \%$ had no synteny. The BGC analysis also indicated that gene duplication only happened for core genes. For example, in PKS Clade 4, S. oryzae Cluster42 had good synteny with S. brachiariae Cluster7, while the other three $S$. oryzae clusters had poor synteny and only had similar PKS genes. This suggested that gene duplication is responsible for the formation of new BGCs in the Sarocladium genus. Lind et al. examined BGC variation in 66 strains of A. fumigatus. They found that the BGCs were highly distinct even in strains belong to one species and identified five different types of variants [25]. Our results are consistent with those of Lind's study, and based on their theory, BGCs variants between $S$. brachiariae and $S$. oryzae should mainly belong be single nucleotide polymorphisms, indels, whole gene cluster polymorphisms, and gene content polymorphisms. BGC variants result in changes in the corresponding metabolites; therefore, we speculated that secondary metabolome of $S$. brachiariae differ markedly from that of $S$. oryzae. Thus, the $S$. brachiariae genome might encode protein responsible for the synthesis of a variety of unknown-function secondary metabolites, possibly related to its adaptation to an endophytic lifestyle and antifungal activity.

\section{Conclusion}

The Sarocladium genus contains different kinds of fungi, such as phytopathogens, endophytes, opportunistic 
human pathogens, and mycoparasites However, to date, only the genome of the phytopathogen $S$. oryzae has been sequenced and published [6]. In the present study, we reported the gapless whole genome sequence of $S$. brachiariae, the first genome sequenced from an endophytic fungus of the Sarocladium genus. The whole genome sequencing and de novo assembly revealed that the genome of $S$. brachiariae comprises $31.86 \mathrm{Mb}$ and the $\mathrm{G}+\mathrm{C}$ content is $52.04 \%$. Comparative genomics analysis revealed that $S$. brachiaria had $14.9 \%$ more plant cell wall degradation related CAZymes to $S$. oryzae, and $33.3 \%$ more fungal cell wall degradation related CAZymes. The expanded fungal cell wall degradation related CAZymes might be the reason why $S$. brachiaria shows antifungal activity. Based on the antiSMASH analysis result, we identified a contact biosynthetic gene cluster for helvolic acid in S. oryzae for the first time. However, we found no gene cluster related to helvolic acid biosynthesis or a gene encoding a cytochrome P450 belonging to the CYP5081 family, which are necessary for helvolic acid biosynthesis, in the genome of S. brachiariae. This indicated that the endophytic fungus $S$. brachiariae could not synthesize the phytotoxin helvolic acid. Including the missing helvolic acid BGC, S. brachiaria had seven fewer terpene gene clusters compared with those in S. oryzae, which might be an adaptation method for its endophytic lifestyle. Synteny analysis of PKS, NRPS, and PKS/NRPS gene clusters between $S$. brachiariae and S. oryzae revealed that just $37.5 \%$ of these clusters have good synteny, while $63.5 \%$ have no or poor synteny. This indicated that the $S$. brachiariae could potentially synthesize a variety of unknownfunction secondary metabolites, which may play an important role in adaptation to its endophytic lifestyle and antifungal activity.

These findings will form the basis for further experimental studies on the endophytic fungus S. brachiariae.

\section{Methods}

Culture and genomic DNA and RNA extraction

Endophytic fungi Sarocladium brachiariae HND5 (China General Microbiological Culture Collection Center, CGMCC 2192) was isolated and maintained in our laboratory. Fungi was grown on potato dextrose agar medium and incubated at $28{ }^{\circ} \mathrm{C}$ for 7 days. Mycelia were harvested and DNA was extracted from grounded mycelia using a modified cetyltrimethylammonium bromide (CTAB) method, as described previously [58]. For RNA extraction, mycelia were harvested from 7-day-old PDA plate and potato dextrose broth culture incubated for 7 days. Mycelia was immediately flash frozen in liquid nitrogen and TRNzol Universal RNA extraction kit (Tiangen, Beijing) was used for total RNA extraction. Agarose gel electrophoresis, a NanoDrop 1000 spectrophotometer (Thermo, USA), and a Qubit fluorometer (Thermo, USA) were used to analyze the integrity, quality, and concentration of total DNA and RNA, respectively.

\section{Genome sequencing and assembly}

DNA was randomly sheared into fragments with an average size of $20 \mathrm{~kb}$ using a Covaris g-TUBE. DNA damage and the ends of the sheared DNA were then repaired. SMRTbell templates were obtained by ligating the blunt hairpin adapters to the ends of the repaired fragments, followed by the addition of exonuclease to remove failed ligation products. Before annealing the sequencing primer and binding the polymerase to SMRTbell templates, the quality of library was assessed using an Agilent 2100 Bioanalyzer High Sensitivity Kit (Agilent, USA). SMRT cells were sequenced using the PacBio RS II sequencing platform (Pacific Biosciences, Nextomics Biosciences, Co., Ltd., Wuhan). After filtering out the sequencing adapters and low-quality sequences, clean data (filtered reads: 4.92G, sequencing depth: 191 $\times$ ) were obtained and then assembled using CANU (https://canu.readthedocs.io/en/latest/\#) with default parameters [59]. The assembly result was adjusted using Arrow [27] and the integrity of assembly was evaluated using BUSCO [60].

\section{RNA sequencing and data analysis}

RNA sample was sequenced with paired-end, 150-bp reads on Illumina novaseq 6000 system (Nextomics Biosciences, Co., Ltd., Wuhan). The sequencing reads were mapped to the Sarocladium brachiariae genome using the TopHat 2.1.1 with default parameters [61].

\section{Genome annotation}

Protein coding genes were annotated using a combination of two different methods: (1) Augustus and Genscan were used to de novo predict protein coding genes by constructing models; (2) GeneWise was used to predict protein coding genes by homology analysis with known protein sequences from related species related species (Magnaporthe oryzae, Fusarium oxysporum, Pochonia chlamydosporia, Claviceps purpurea and Verticillium dahlia) [62, 63]. EVidenceModeler (EVM) was then used to compute the weighted consensus gene structure annotations [64]. After removing genes with transposable elements using TransposonPSI [65], we obtained the final gene sets.

Multiple databases, including Swiss-Prot, NR, KEGG, and COG were used to make functional annotations for the predicted gene models, using BlastP with E-values $\leq 1^{e-5}$ [66-68]. BLAST searching against the Rfam database was used to predict non-coding RNAs, such as rRNAs, snRNAs, and miRNAs [69]. RNAmmer and 
tRNAscan-SE were also used to predict rRNAs and tRNAs, respectively [70, 71].

\section{Repetitive sequences analysis}

Repetitive sequences were identified and analyzed using different methods. Four computer applications were used to identify transposable elements, including the databasebased software RepeatProteinMasker and RepeatMasker, and two de novo pieces of software, RepeatModeler (http://repeatmasker.org/RepeatModeler/) and LTRfinder [72-74]. Tandem Repeats Finder was used to analyze tandem repeat sequences and MIcroSAtellite was used to detect the microsatellite DNA (1-6 bp) $[75,76]$.

\section{Analysis of orthologous gene families in Sordariomycetes fungi}

Gene families were analyzed using orthoMCL [77] (Evalues $\leq 1^{e-5}$ ) by comparing proteins from $S$. brachiariae with those from other Sordariomycetes fungi: $S$. oryzae (GenBank: GCA_001972265.1), A. chrysogenum (GenBank: GCA_000769265.1), F. graminearum (GenBank: GCA_000 240135.3), F. oxysporum (GenBank: GCA_000149955.2), and M. oryzae (GenBank: GCA_000002495.2).

\section{Phylogenetic analysis and synteny analysis}

Based on the orthologous gene families analysis, single copy orthologous gene groups were chosen for further phylogenetic analysis. Gblocks [78] (with default parameters) was used to remove divergence and ambiguously aligned blocks from the alignment to obtain a better CDS file. The maximum-likelihood tree was constructed using RaxML with the GTRGAMMA model and 100 bootstrap replicates to infer the phylogenetic relationship of $S$. brachiariae to other Sordariomycetes fungi $(S$. oryzae, A. chrysogenum, F. graminearum, F. oxysporum, Coniochaeta ligniaria (GenBank: GCA_001879275.1), Verticillium dahlia (GenBank: GCA_000952015.1), and Pochonia chlamydosporia (GenBank: GCA_001653235.2) [30]. MUMmer software was used to perform the genome-wide synteny analysis, and synteny dot-plot were generated using mummerplot [32].

\section{Pathogenicity genes analysis}

The S. brachiariae proteome was used to make a BLASTP search (E-values $\leq 1^{e-5}$ ) against the PathogenHost Interaction (PHI) (http://www.phi-base.org) database to identify fungal pathogenicity genes [79].

\section{Carbohydrate-active (CAZy) enzymes analysis}

The S. brachiariae proteins were used to make a BLASTP search(E-values $\leq 1 \mathrm{e}-5$ ) against the Carbohydrate Active Enzymes database (http://www.cazy.org/) [7]. The results were catalyzed like Glycoside Hydrolases (GHs), Glycosyl Transferases (GTs), Polysaccharide
Lyases (PLs), Carbohydrate Esterases (CEs), Carbohydrate-Binding Modules (CBMs), and Auxillary Activities (AAs) as described in CAZy database.

\section{Cytochrome P450 analysis}

The cytochrome $\mathrm{P} 450$ gene family classification (Evalues $\leq 1^{e-5}$, identity $>40 \%$ ) in S. brachiariae was performed using the CYtochrome P450 Engineering Database (version6.0)(https://cyped.biocatnet.de/).

\section{Secretome analysis}

The prediction of the refined S. brachiariae and S. ory$z a e$ secretome was based on the procedure described by Brown and colleagues for Fusarium graminearum [80]. SignalP (http://www.cbs.dtu.dk/services/SignalP/) was used to predict signal peptides and cleavage sites of $S$. brachiariae and S. oryzae proteins [81]. Proteins with a Singal P D-score $=\mathrm{Y}$ were analyzed for subcellular location with Target P v1.1 (http://www.cbs.dtu.dk/services/ TargetP/) [82]. And proteins with a Target $\mathrm{P}$ Loc $=\mathrm{S}$ were scanned for transmembrane spanning regions using TMHMM (TMHMM v2.0; http://www.cbs.dtu.dk/services/TMHMM/) and all proteins with $0 \mathrm{TMs}$ or $1 \mathrm{TM}$, if located in the predicted $\mathrm{N}$-terminal signal peptide, were kept. Proteins with glycosylphosphatidylinositol (GPI) anchor were predicted by big-PI [83] (http://mendel.imp.ac.at/gpi/gpi_server.html). Localization of the remaining proteins without GPI-anchor were predicted with ProtComp using the LocDB and PotLocDB databases (ProtComp v9.0; http://www.softberry.com/berry. phtml? topic $=$ protcompan\&group $=$ programs\&subgrous $=$ proloc) and proteins predicted as extracellular or unknown were kept for next analysis. Proteins with no methionine at start or mature peptide less than 20 amino acids were discarded. WolfPSort (https://wolfpsort.hgc. $\mathrm{jp} /$ ) [84] were used to analyze the remaining proteins and proteins with extracellular score $>17$ were kept in the final secretome databases.

\section{Secondary metabolite gene cluster analysis}

Putative polyketide synthases (PKS), non-ribosomal peptide synthases (NRPS) genes, PKS-NRPS hybrids, and their modules of different domains were identified via searching in the antiSMASH database (fungal version) (https://fungismash.secondarymetabolites.org/) with default settings [85].

A Maximum Likelihood tree was generated with amino acid sequences of $\mathrm{A}$ or KS domains using MEGA version 6.0 with the Wheland and Goldman (WAG) mode [86]. A domains and KS domains sequences used for Maximum Likelihood tree construction were listed in Additional file 6 and Additional file 7. GATA was used to perform synteny analysis of gene clusters, with default settings [87]. Genes found in PKS, NRPS and PKS/NRPS gene clusters 
were annotated based on BLASTN search results and results were listed in Additional file 4 (S. brachiariae) and Additional file 5 (S. oryzae).

\section{PCR validation of in silico gene prediction result of $S$. brachiariae}

22 genes were picked from 22 secondary gene clusters of S. brachiariae for PCR validation of its in silico gene prediction result. These genes and their primers were listed in Additional file 2: Table S9. TaKaRa Taq DNA polymerase (Takara Biomedical Technology, Beijing) was used for PCR amplification according to product protocol. A touchdown PCR program was used for PCR amplification: $95^{\circ} \mathrm{C} 2 \mathrm{~min} ; 95^{\circ} \mathrm{C} 20 \mathrm{~s} ; 65^{\circ} \mathrm{C} 20 \mathrm{~s}\left(-1{ }^{\circ} \mathrm{C}\right.$ per cycle, ramp $2{ }^{\circ} \mathrm{C}$ ); $70^{\circ} \mathrm{C}, 40 \mathrm{~s}$; go to step 2 for 20 cycles; $70^{\circ} \mathrm{C}, 10 \mathrm{~min} .1 \%$ agarose gel electrophoresis was used for PCR products analysis and target DNA bands were cut and purified for Sanger sequencing (Huada Gene Technology, Shenzhen).

\section{Supplementary information}

Supplementary information accompanies this paper at https://doi.org/10. 1186/s12864-019-6095-1.

Additional file 1: Figure S1. Agarose gel electrophoretogram of PCR amplification products of 22 genes chosen for validation of in silico gene prediction result of S. brachiariae. Line 1-22: WHWLZ0913, WHWLZ1690, WHWLZ1900, WHWLZ1931, WHWLZ2227, WHWLZ2744, WHWLZ2917, WHWLZ4323, WHWLZ5125, WHWLZ5511, WHWLZ5935, WHWLZ6101, WHWLZ6305, WHWLZ7443, WHWLZ7561, WHWLZ7986, WHWLZ8202, WHWLZ8477, WHWLZ9395, WHWLZ9449, WHWLZ9463 and WHWLZ9474.

Additional file 2: Table S1.1. BUSCO analysis of S. brachiariae scaffolds assembly; Table S1.2. Statistics of RNAseq data; Table S1.3. Mapping rate of NGS data; Table 2. RNA statistics of S. brachiariae; Table S3. Repeative sequence statistic of S. brachiariae; Table S4. Gene annotation statistic of S. brachiariae; Table S5.1. KOG distribution of predicted proteins from S. brachiariae; Table S5.2. KOG distribution of predicted proteins from S. oryzae; Table S6.1. Secreated proteins of S. brachiariae and S. oryzae; Table S6.2. CAZymes distribution of predicted proteins from S. brachiariae; Table S6.3. CAZymes distribution of predicted proteins from S. oryzae; Table S7.1. SM Clusters coordinates of S. brachiariae scaffolds assembly; Table S7.2. SM Clusters coordinates of S. oryzae scaffolds assembly; Table S8. Cytochrome P450 distribution of predicted proteins from S. brachiariae; Table S9. Genes chosen for PCR validation of in silico gene prediction result of S. brachiariae and primers design.

Additional file 3: Table S1. Helvolic acid biosynthetic gene cluster found in S. oryzae; Table S2. Possible proteins involved in helvolic acid biosynthesis found in S. brachiariae.

Additional file 4. Annotation (BLASTp) of genes in PKS, NRPS and PKS/ NRPS gene clusters of S. brachiariae.

Additional file 5. Annotation (BLASTp) of genes in PKS, NRPS and PKS/ NRPS gene clusters of S. oryzae.

Additional file 6. A domain sequences used for phylogenetic analysis. Additional file 7. KS domain sequences used for phylogenetic analysis.

Additional file $\mathbf{8}$. Sanger sequencing results of the PCR products of the 22 genes chosen for PCR validation of in silico gene prediction result of S. brachiariae. (TXT $20 \mathrm{~kb}$ )

\section{Abbreviations}

A: Adenylation; ACP: Acyl Carrier Protein; antiSMASH: Antibiotics \& Secondary Metabolite Analysis Shell; BGC: Biosynthetic Gene Cluster; C: Condensation;
CAZyme: Carbohydrate-active Enzyme; CE: Carbohydrate Esterase: CWEDs: Cell Wall-degrading Enzymes; CYP: Cytochrome P450; DH: Dehydratase; GH: Glycoside Hydrolase; KR: Ketoreductase; KS: Ketosynthase; KSTD: 3-ketosteroid- $\Delta$ 1-dehydrogenase; MAT: MalonylCoA:acyl Carrier Protein Transacylase; NRPS: Non-ribosomal Peptide Synthetases; OSC: Oxidosqualene Cyclase; PKS: Polyketide Synthases; PL: Polysaccharide Lyases; SDR: Short-chain dehydrogenase/reductase; SM: Secondary Metabolite; T: Thiolation; TC: Terpene Cyclases

\section{Acknowledgements}

We would like to thank JY Wu (Genedenovo Biotechnology Co., Ltd., Guangzhou) and Pan Xu (Diggers Biotechnology Co., Ltd., Wuhan) for the support on data analysis.

\section{Authors' contributions}

$Y Y$ performed sequence, bioinformatic analyses and wrote the main manuscript text, $\mathrm{XL}$ performed the phylogenetic analysis, JC performed genomic DNA extraction, YC, BL, ZG and GH made a critical revision of the manuscript. All authors read and approved the final manuscript.

\section{Funding}

This study was supported in part by grants from Hainan Provincial Natural Science Foundation of China, 319QN268; National Key R\&D Program of China, 2017YFD0202100; Central Public-interest Scientific Institution Basal Research Fund for Chinese Academy of Tropical Agricultural Sciences, 1630042019006. The funding bodies had no roles in the design of the study and collection, analysis, and interpretation of data and in writing the manuscript.

\section{Availability of data and materials}

The genome assembly of S. brachiariae generated and analyzed during this studyhas been deposited at DDBJ/ENA/GenBank under the accession RQPE00000000. The version described in this paper is version RQPE01000000. The raw sequence reads are deposited in NCBI SRA database under accession number SRR8202370.

The raw sequence reads of RNAseq are deposited in NCBI SRA database under accession number SRR9289321.

\section{Ethics approval and consent to participate}

Not applicable.

\section{Consent for publication}

Not applicable.

\section{Competing interests}

The authors declare that they have no competing interests.

\section{Author details}

${ }^{1}$ Environment and Plant Protection Institute, Chinese Academy of Tropical Agricultural Sciences, 4 Xueyuan Road, Haikou 571101, China. ${ }^{2}$ Institute of Tropical Bioscience and Biotechnology, Chinese Academy of Tropical Agricultural Sciences, 4 Xueyuan Road, Haikou 571101, China.

Received: 26 November 2018 Accepted: 10 September 2019 Published online: 28 October 2019

\section{References}

1. Gams W, Hawksworth DL: Identity of Acrocylindrium oryzae Sawada and a similar fungus causing sheath-rot of rice. Kavaka 1975.

2. Giraldo A, Gené J, Sutton D, Madrid H, De Hoog G, Cano J, Decock C, Crous PW, Guarro J: Phylogeny of Sarocladium (Hypocreales). Persoonia, 2015, 34:10

3. Summerbell R, Gueidan C, Schroers H, De Hoog G, Starink M, Rosete YA Guarro J, Scott J. Acremonium phylogenetic overview and revision of Gliomastix, Sarocladium, and Trichothecium. Stud Mycol. 2011;68:139-62.

4. Liu X, Guo Z, Huang G. Sarocladium brachiariae sp. nov., an endophytic fungus isolated from Brachiaria brizantha. Mycosphere. 2017;8:827-34.

5. Huang GX, Shi T, Liu XB Dai YK, Cai JM and Lin CH: GFP-tagging and antagonistic activity of the engineered strains of Brachiaria brizantha endophytic fungus HND5. Chinese Journal of Biological Control 2010, 26: 320-326. (In Chinese). 
6. Hittalmani S, Mahesh HB, Mahadevaiah C, Prasannakumar MK. De novo genome assembly and annotation of rice sheath rot fungus Sarocladium oryzae reveals genes involved in Helvolic acid and Cerulenin biosynthesis pathways. BMC Genomics. 2016;17(1):271.

7. Lombard V, Golaconda Ramulu H, Drula E, Coutinho PM, Henrissat B. The carbohydrate-active enzymes database (CAZy) in 2013. Nucleic Acids Res. 2013;42(D1):D490-5.

8. Kubicek CP, Starr TL, Glass NL. Plant cell wall-degrading enzymes and their secretion in plant-pathogenic fungi. Annu Rev Phytopathol. 2014;52:427-51.

9. Underwood W. The plant cell wall: a dynamic barrier against pathogen invasion. Front Plant Sci. 2012;3:85.

10. Zhao Z, Liu H, Wang C, Xu J-R. Comparative analysis of fungal genomes reveals different plant cell wall degrading capacity in fungi. BMC Genomics. 2013;14(1):274.

11. van den Brink J, de Vries RP. Fungal enzyme sets for plant polysaccharide degradation. Appl Microbiol Biotechnol. 2011;91(6):1477.

12. Latgé JP. The cell wall: a carbohydrate Armour for the fungal cell. Mol Microbiol. 2007;66(2):279-90.

13. Langner T, Göhre V. Fungal chitinases: function, regulation, and potential roles in plant/pathogen interactions. Curr Genet. 2016;62(2):243-54.

14. Gruber S, Seidl-Seiboth V. Self versus non-self: fungal cell wall degradation in Trichoderma. Microbiology. 2012;158(1):26-34.

15. Keller NP, Turner G, Bennett JW. Fungal secondary metabolism—from biochemistry to genomics. Nat Rev Microbiol. 2005;3(12):937.

16. Sakthivel N, Amudha R, Muthukrishnan S. Production of phytotoxic metabolites by Sarocladium oryzae. Mycol Res. 2002;106(5):609-14.

17. Ayyadurai N, Kirubakaran SI, Srisha S, Sakthivel N. Biological and molecular variability of Sarocladium oryzae, the sheath rot pathogen of rice (Oryza sativa L.). Curr Microbiol. 2005;50(6):319-23.

18. Omura $\mathrm{S}$. The antibiotic cerulenin, a novel tool for biochemistry as an inhibitor of fatty acid synthesis. Bacteriol Rev. 1976;40(3):681.

19. Mitsuguchi H, Seshime $Y$, Fujii I, Shibuya M, Ebizuka Y, Kushiro T. Biosynthesis of steroidal antibiotic fusidanes: functional analysis of oxidosqualene cyclase and subsequent tailoring enzymes from Aspergillus fumigatus. J Am Chem Soc. 2009;131(18):6402-11.

20. Gao Q, Jin K, Ying S-H, Zhang Y, Xiao G, Shang Y, Duan Z, Hu X, Xie X-Q, Zhou $G$. Genome sequencing and comparative transcriptomics of the model entomopathogenic fungi Metarhizium anisopliae and M. acridum. PLoS Genet. 2011;7(1):e1001264.

21. Marahiel MA. A structural model for multimodular NRPS assembly lines. Nat Prod Rep. 2016;33(2):136

22. Zabala AO, Chooi Y-H, Choi MS, Lin H-C, Tang Y. Fungal polyketide synthase product chain-length control by partnering thiohydrolase. ACS Chem Biol. 2014:9(7):1576-86.

23. Bushley KE, Turgeon BG. Phylogenomics reveals subfamilies of fungal nonribosomal peptide synthetases and their evolutionary relationships. BMC Evol Biol. 2010;10(1):26.

24. Baker SE, Perrone G, Richardson NM, Gallo A, Kubicek CP. Phylogenomic analysis of polyketide synthase-encoding genes in Trichoderma. Microbiology. 2012;158(1):147-54

25. Lind AL, Wisecaver JH, Lameiras C, Wiemann P, Palmer JM, Keller NP Rodrigues F, Goldman GH, Rokas A. Drivers of genetic diversity in secondary metabolic gene clusters within a fungal species. PLoS Biol. 2017;15(11): e2003583.

26. Choque E, Klopp C, Valiere S, Raynal J, Mathieu F. Whole-genome sequencing of Aspergillus tubingensis G131 and overview of its secondary metabolism potential. BMC Genomics. 2018;19(2):200.

27. Chin CS, Alexander DH, Marks P, Klammer AA, Drake J, Heiner C, Clum A, Copeland A, Huddleston J, Eichler EE, et al. Nonhybrid, finished microbial genome assemblies from long-read SMRT sequencing data. Nat Methods. 2013;10(6):563-9.

28. Mohanta TK, Bae H. The diversity of fungal genome. Biological Procedures Online. 2015;17(1):1-9.

29. Gabaldón T, Koonin EV. Functional and evolutionary implications of gene orthology. Nat Rev Genet. 2013;14(5):360.

30. Stamatakis A. RAxML-VI-HPC: maximum likelihood-based phylogenetic analyses with thousands of taxa and mixed models. Bioinformatics. 2006; 22(21):2688-90.

31. Tatusov RL, Fedorova ND, Jackson JD, Jacobs AR, Kiryutin B, Koonin EV, Krylov DM, Mazumder R, Mekhedov SL, Nikolskaya AN. The COG database: an updated version includes eukaryotes. BMC bioinformatics. 2003;4(1):41.
32. Kurtz S, Phillippy A, Delcher AL, Smoot M, Shumway M, Antonescu C, Salzberg SL. Versatile and open software for comparing large genomes. Genome Biol. 2004;5(2):R12.

33. Keller NP. Translating biosynthetic gene clusters into fungal armor and weaponry. Nat Chem Biol. 2015;11(9):671.

34. Tschen JS-M, Chen L-L, Hsieh S-T, Wu T-S. Isolation and phytotoxic effects of helvolic acid from plant pathogenic fungus Sarocladium oryzae. Botanical Bulletin of Academia Sinica. 1997;38.

35. Lv J-M, Hu D, Gao H, Kushiro T, Awakawa T, Chen G-D, Wang C-X, Abe I, Yao X-S. Biosynthesis of helvolic acid and identification of an unusual C-4-demethylation process distinct from sterol biosynthesis. Nat Commun. 2017;8(1):1644.

36. Kroken S, Glass NL, Taylor JW, Yoder O, Turgeon BG. Phylogenomic analysis of type I polyketide synthase genes in pathogenic and saprobic ascomycetes. Proc Natl Acad Sci. 2003;100(26):15670-5.

37. Etienne KA, Roe CC, Smith RM, Vallabhaneni S, Duarte C, Escandón P, Castañeda E, Gómez BL, de Bedout C, López LF. Whole-genome sequencing to determine origin of multinational outbreak of Sarocladium kiliense bloodstream infections. Emerg Infect Dis. 2016;22(3):476.

38. Dovana F, Mucciarelli M, Mascarello M, Fusconi A. In vitro morphogenesis of Arabidopsis to search for novel endophytic fungi modulating plant growth. PLoS One. 2015:10(12):e0143353.

39. Potshangbam M, Devi SI, Sahoo D, Strobel GA. Functional characterization of endophytic fungal community associated with Oryza sativa L. and Zea mays L. Front Microbiol. 2017;8:325.

40. Donald T, Shoshannah R, Deyrup ST, Gloer JB. A protective endophyte of maize: Acremonium zeae antibiotics inhibitory to Aspergillus flavus and Fusarium verticillioides. Mycol Res. 2005;109(5):610-8.

41. Knogge W. Fungal infection of plants. Plant Cell. 1996;8(10):1711.

42. Wu W, Davis RW, Tran-Gyamfi MB, Kuo A, LaButti K, Mihaltcheva S, Hundley H, Chovatia M, Lindquist E, Barry K. Characterization of four endophytic fungi as potential consolidated bioprocessing hosts for conversion of lignocellulose into advanced biofuels. Appl Microbiol Biotechnol. 2017;101(6):2603-18.

43. Aranda-Martinez A, Lenfant N, Escudero N, Zavala-Gonzalez EA, Henrissat B, Lopez-Llorca LV. CAZyme content of Pochonia chlamydosporia reflects that chitin and chitosan modification are involved in nematode parasitism. Environ Microbiol. 2016;18(11):4200-15.

44. Xu X, He Q, Chen C: Differential Communications between Fungi and Host Plants Revealed by Secretome Analysis of Phylogenetically Related Endophytic and Pathogenic Fungi. 2016, 11(9):e0163368.

45. Cortes-Tolalpa L, Norder J, van Elsas JD, Falcao Salles J. Halotolerant microbial consortia able to degrade highly recalcitrant plant biomass substrate. Appl Microbiol Biotechnol. 2018;102(6):2913-27.

46. Longoni P, Rodolfi M, Pantaleoni L, Doria E, Concia L, Picco AM, Cella R. Functional analysis of the degradation of cellulosic substrates by a Chaetomium globosum endophytic isolate. Appl Environ Microbiol. 2012;78(10):3693-705.

47. Ownley BH, Gwinn KD, Vega FE. Endophytic fungal entomopathogens with activity against plant pathogens: ecology and evolution. BioControl. 2010; 55(1):113-28.

48. Berestetskiy A. A review of fungal phytotoxins: from basic studies to practical use. Appl Biochem Microbiol. 2008;44(5):453.

49. Bigirimana VP, Hua GK, Nyamangyoku OI, Höfte M. Rice sheath rot: an emerging ubiquitous destructive disease complex. Front Plant Sci. 2015;6:1066.

50. Zhang J-S, Lei J-P, Wei G-Q, Chen H, Ma C-Y, Jiang H-Z. Natural fatty acid synthase inhibitors as potent therapeutic agents for cancers: a review. Pharm Biol. 2016:54(9):1919-25.

51. Albalat R, Cañestro C. Evolution by gene loss. Nat Rev Genet. 2016;17(7):379.

52. Xia C, Wang M, Yin C, Cornejo OE, Hulbert SH, Chen X. Genomic insights into host adaptation between the wheat stripe rust pathogen (Puccinia striiformis $\mathrm{f}$. sp. tritici) and the barley stripe rust pathogen (Puccinia striiformis f. sp. hordei). BMC Genomics. 2018;19(1):664.

53. Chuma I, Isobe C, Hotta Y, Ibaragi K, Futamata N, Kusaba M, Yoshida K, Terauchi R, Fujita Y, Nakayashiki H. Multiple translocation of the AVR-Pita effector gene among chromosomes of the rice blast fungus Magnaporthe oryzae and related species. PLoS Pathog. 2011;7(7):e1002147.

54. Gout L, Kuhn ML, Vincenot L, Bernard-Samain S, Cattolico L, Barbetti M, Moreno-Rico O, Balesdent MH, Rouxel T. Genome structure impacts molecular evolution at the AvrLm1 avirulence locus of the plant pathogen Leptosphaeria maculans. Environ Microbiol. 2007:9(12):2978-92.

55. Schürch S, Linde CC, Knogge W, Jackson LF, McDonald BA. Molecular population genetic analysis differentiates two virulence mechanisms of the fungal avirulence gene NIP1. Mol Plant-Microbe Interact. 2004;17(10):1114-25. 
56. Spatafora JW, Bushley KE. Phylogenomics and evolution of secondary metabolism in plant-associated fungi. Curr Opin Plant Biol. 2015;26:37-44.

57. Carbone I, Ramirez-Prado JH, Jakobek JL, Horn BW. Gene duplication, modularity and adaptation in the evolution of the aflatoxin gene cluster. BMC Evol Biol. 2007;7(1):111.

58. Kim JS, Jun BK, Kim SH. Simple and reliable DNA extraction method for the dark pigmented fungus, Cercospora sojina. The Plant Pathology Journal. 2010;26(3):289-92.

59. Koren S, Walenz BP, Berlin K, Miller JR, Bergman NH, Phillippy AM. Canu: scalable and accurate long-read assembly via adaptive k-mer weighting and repeat separation. Genome Res. 2017;27(5):722-36.

60. Simão FA, Waterhouse RM, loannidis P, Kriventseva EV, Zdobnov EM BUSCO: assessing genome assembly and annotation completeness with single-copy orthologs. Bioinformatics. 2015;31(19):3210-2.

61. Kim D, Pertea G, Trapnell C, Pimentel H, Kelley R, Salzberg SL. TopHat2: accurate alignment of transcriptomes in the presence of insertions, deletions and gene fusions. Genome Biol. 2013;14(4):R36.

62. Birney E, Durbin R. Using GeneWise in the Drosophila annotation experiment. Genome Res. 2000;10(4):547-8.

63. Stanke M, Waack S. Gene prediction with a hidden Markov model and a new intron submodel. Bioinformatics. 2003;19(suppl_2):ii215-25.

64. Haas BJ, Salzberg SL, Zhu W, Pertea M, Allen JE, Orvis J, White O, Buell CR, Wortman JR. Automated eukaryotic gene structure annotation using EVidenceModeler and the program to assemble spliced alignments. Genome Biol. 2008;9(1):R7.

65. Yagi M, Kosugi S, Hirakawa H, Ohmiya A, Tanase K, Harada T, Kishimoto K, Nakayama M, Ichimura K, Onozaki T. Sequence analysis of the genome of carnation (Dianthus caryophyllus L.). DNA Res. 2013;21(3):231-41.

66. Kanehisa M, Goto S, Kawashima S, Okuno Y, Hattori M. The KEGG resource for deciphering the genome. Nucleic Acids Res. 2004;32(suppl_ 1):D277-80

67. Tatusov RL, Galperin MY, Natale DA, Koonin EV. The COG database: a tool for genome-scale analysis of protein functions and evolution. Nucleic Acids Res. 2000;28(1):33-6.

68. Altschul SF, Gish W, Miller W, Myers EW, Lipman DJ. Basic local alignment search tool. J Mol Biol. 1990;215(3):403-10.

69. Griffiths-Jones S, Moxon S, Marshall M, Khanna A, Eddy SR, Bateman A. Rfam: annotating non-coding RNAs in complete genomes. Nucleic Acids Res. 2005;33(suppl_1):D121-4.

70. Lagesen $\mathrm{K}$, Hallin $\mathrm{P}$, Rødland EA, Stærfeldt H-H, Rognes T, Ussery DW. RNAmmer: consistent and rapid annotation of ribosomal RNA genes. Nucleic Acids Res. 2007;35(9):3100-8.

71. Lowe TM, Eddy SR. tRNAscan-SE: a program for improved detection of transfer RNA genes in genomic sequence. Nucleic Acids Res. 1997;25(5):955.

72. Allred DB, Cheng A, Sarikaya M, Baneyx F, Schwartz DT. Three-dimensional architecture of inorganic nanoarrays electrodeposited through a surfacelayer protein mask. Nano Lett. 2008;8(5):1434-8.

73. Bedell JA, Korf I, Gish W. MaskerAid: a performance enhancement to RepeatMasker. Bioinformatics. 2000;16(11):1040-1.

74. Xu Z, Wang H. LTR_FINDER: an efficient tool for the prediction of full-length LTR retrotransposons. Nucleic Acids Res. 2007;35(suppl_2):W265-8.

75. Benson G. Tandem repeats finder: a program to analyze DNA sequences. Nucleic Acids Res. 1999:27(2):573.

76. Thiel T, Michalek W, Varshney R, Graner A. Exploiting EST databases for the development and characterization of gene-derived SSR-markers in barley (Hordeum vulgare L.). Theor Appl Genet. 2003;106(3):411-22.

77. Li L, Stoeckert CJ Jr, Roos DS. OrthoMCL: identification of ortholog groups for eukaryotic genomes. Genome Res. 2003;13(9):2178-89.

78. Castresana J. Selection of conserved blocks from multiple alignments for their use in phylogenetic analysis. Mol Biol Evol. 2000;17(4):540-52.

79. Urban M, Pant R, Raghunath A, Irvine AG, Pedro H, Hammond-Kosack KE. The pathogen-host interactions database (PHI-base): additions and future developments. Nucleic Acids Res. 2014;43(D1):D645-55.

80. Brown NA, Antoniw J, Hammond-Kosack KE. The predicted secretome of the plant pathogenic fungus Fusarium graminearum: a refined comparative analysis. PLoS One. 2012;7(4):e33731.

81. Emanuelsson O, Brunak S, Von Heijne G, Nielsen $\mathrm{H}$. Locating proteins in the cell using TargetP, SignalP and related tools. Nat Protoc. 2007;2(4):953.

82. Emanuelsson O, Nielsen H, Brunak S, Von Heijne G. Predicting subcellular localization of proteins based on their $\mathrm{N}$-terminal amino acid sequence. J Mol Biol. 2000;300(4):1005-16.
83. Eisenhaber B, Bork P, Eisenhaber F. Prediction of potential GPI-modification sites in proprotein sequences. J Mol Biol. 1999:292(3):741-58.

84. Horton P, Park KJ, Obayashi T, Fujita N, Harada H, Adams-Collier CJ, Nakai K. WoLF PSORT: protein localization predictor. Nucleic Acids Res. 2007;35(Web Server):W585-7.

85. Weber T, Blin K, Duddela S, Krug D, Kim HU, Bruccoleri R, Lee SY, Fischbach MA, Müller R, Wohlleben W. antiSMASH 3.0-a comprehensive resource for the genome mining of biosynthetic gene clusters. Nucleic Acids Res. 2015; 43(W1):W237-43.

86. Tamura K, Stecher G, Peterson D, Filipski A, Kumar S. MEGA6: molecular evolutionary genetics analysis version 6.0. Mol Biol Evol. 2013;30(12):2725-9.

87. Nix DA, Eisen MB. GATA: a graphic alignment tool for comparative sequence analysis. BMC bioinformatics. 2005;6(1):9.

\section{Publisher's Note}

Springer Nature remains neutral with regard to jurisdictional claims in published maps and institutional affiliations.

\section{Ready to submit your research? Choose BMC and benefit from:}

- fast, convenient online submission

- thorough peer review by experienced researchers in your field

- rapid publication on acceptance

- support for research data, including large and complex data types

- gold Open Access which fosters wider collaboration and increased citations

- maximum visibility for your research: over $100 \mathrm{M}$ website views per year

At $\mathrm{BMC}$, research is always in progress.

Learn more biomedcentral.com/submissions 